\title{
IMPLICIT BEM FORMULATIONS FOR USUAL AND SENSITIVITY PROBLEMS IN ELASTO- PLASTICITY USING THE CONSISTENT TANGENT OPERATOR CONCEPT
}

\author{
MARC BONNET \\ Laboratoire de Mécanique des Solides, CNRS URA 317, Centre Commun Polytechnique, \\ Mines, Ponts et Chausées, Ecole Polytechnique, Palaiseau, France \\ and \\ SUBRATA MUK HERJEE \\ Depastment of Theoretical and Applied Mechanics, Cornell University, Ithaca, NY, U.S.A.
}

\begin{abstract}
This paper presents boundary element method (BEM) formulations for usual and sensitivity problems in (small strain) elasto-plasticity using the concept of the local consistent tangent operator (CTO). "Usual" problems here refer to analysis of nonlinear problems in structural and solid continua, for which Simo and Taylor first proposed the use of the CTO within the context of the finite element method (FEM). A new implicit BEM scheme for such problems, using the CTO, is presented first. A formulation for sensitivity analysis follows. It is shown that the sensitivity of the strain increment, associated with an infinitesimal variation of some design parameter, solves a linear problem which is governed by the (converged value of the) same global CTO as the one that appears in the usual problem. Numerical results for both usual and sensitivity problems are shown for a one-dimensional example. They demonstrate the effectiveness of the present approach. In particular, accurate sensitivities with respect to material parameters (e.g., exponent of the powertype hardlening law) are obtained even with few integration cells and for large load increments.
\end{abstract}

\section{INTRODUCTION}

In this paper, we address two important topics within the context of boundary element method (BEM) analysis of (small strain) elasto-plastic problems. The first is an implicit BEM formulation for usual elasto-plastic analysis and the second is a sensitivity formulation for such problems. Both these formulations involve the consistent tangent operator (CTOsee Simo and Taylor, 1985).

Sensitivity analysis of nonlinear (material and/or geometrical) problems in solid mechanics is an active research area at present. In this context, design sensitivity coefficients (DSCs) are rates of change of response quantities, such as stresses or displacements in a loaded body, with respect to design variables. These design variables could be shape parameters, sizing parameters, boundary conditions, material parameters etc. DSCs are useful in diverse applications, a very important one being optimal design using gradient based optimization algorithms. Such analyses can be applied, for example, to optimal design of certain manufacturing processes.

Currently, the direct differentiation approach (DDA) or the adjoint structure approach (ADA) are popular for accurate sensitivity analysis. Either of these can be applied in conjunction with general purpose numerical methods such as the finite element method (FEM) or the BEM. The FEM has been used for sensitivity analysis of nonlinear problems by, among others, Arora and his co-workers (Arora and Cardoso, 1992; Yao and Arora, 1992a, b), Choi and his co-workers (Choi and Santos, 1987; Santos and Choi, 1988), Haber and his co-workers (Vidal et al., 1991 ; Vidal and Haber, 1993), Kleiber and his co-workers (Kleiber, 199.1 ; Kleiber et al., 1994, 1995), Michaleris et al. (1994) and Badrinarayanan and Zabaras (1996). Haber, Kleiber and their associates were the first to point out that the 
consistent (or algorithmic) tangent operator (CTO) (as opposed to the continuum tangent operator) plays a key role in nonlinear sensitivity analysis. The CTO was originally proposed by Simo and Taylor (1985) for FEM analysis of (usual) nonlinear problems. The sensitivity problem is always linear (even if the usual problem is not) and the global or system matrix related to the CTO is precisely the stiffness matrix for these problems. Use of the CTO, as pointed out by Kleiber, Haber and their associates, provides very accurate numerical results for sensitivities, while other approaches (e.g., using the continuum tangent) might lead to significant errors. These researchers present numerical results for materially nonlinear problems. Michaleris et al. (1994), in a recent paper, present sensitivity formulations for general transient nonlinear coupled problems, together with an accurate numerical procedure for the calculation of the CTO. Badrinarayanan and Zabaras (1995) present a consistent scheme for sensitivity analysis of nonlinear (both material and geometric) problems in solid mechanics. This paper presents very accurate numerical results for sensitivities at the end of a large deformation (extrusion) process.

All the researchers cited above have employed the FEM in order to obtain their numerical results. Mukherjee and his co-workers have been active in solving nonlinear (both material and geometric) sensitivity problems by the BEM. Examples of the work of this group, using the explicit BEM, are Zhang et al. (1992a,b) and Leu and Mukherjee (1993) (see also the forthcoming book: Chandra and Mukherjee, 1996). Wei et al. (1994) have used sensitivities to carry out shape optimal design of an elasto-plastic problem.

Most of the publications on BEM analysis of (usual) nonlinear problems in solid mechanics report on the use of the explicit approach for time integration of the appropriate rate equations. Banerjee and his co-workers (Banerjee, 1994) have presented variable stiffness formulations for such problems. Implicit BEM formulations have been presented by Jin et al. (1989) and Telles and Carrer (1991, 1994). Mukherjee and his co-workers have been interested in implicit sensitivity calculations, using the BEM, during the last few years. Leu and Mukherjee (1994a, b) have presented implicit objective integration schemes for recovery of stress sensitivities at a material point. This work addresses large strain viscoplastic problems but only considers integration of the algorithmic constitutive model (analogous to the radial return algorithm) at a material point. They have coupled this analysis with the BEM (Leu and Mukherjee, 1995) to solve general boundary value problems. The CTO, however, has not been employed in the work by Leu and Mukherjee cited above. It is observed (Leu and Mukherjee, 1995) that stress sensitivities at some material points, at the end of a large deformation process, can exhibit significant numerical errors.

The remedy appears to be an implicit BEM formulation that employs the consistent tangent operator. Within the context of the BEM, this paper presents, for the first time, an implicit scheme that explicitly utilizes the CTO. Small-strain elasto-plastic problems, with isotropic and kinematic hardening behavior, are considered in this paper, but further generalizations present no conceptual difficulties. Next, the corresponding sensitivity formulation is derived. It is shown that the (converged value of) the "global" CTO appears, as expected, as the stiffness matrix for the linear system of equations that govern the sensitivity of the strain increment over a time step. Initial numerical results for sensitivities, using the formulation presented in this paper, are very accurate.

\section{THE GLOBAL CONSISTENT TANGENT OPERATOR USING FEM}

Let $\Omega$ denote the spatial region occupied by the elastic-plastic solid under consideration. Attention is restricted to small-strain, quasistatic loading processes.

\section{Constitutive law}

Let $\mathbf{u}, \boldsymbol{\sigma}$ and $\boldsymbol{\varepsilon}$ denote respectively the displacement, stress tensor and total strain tensor (with $2 \varepsilon=\left(\nabla+\nabla^{\mathrm{T}}\right) \mathbf{u}$, where $\nabla$ is the gradient operator). Following Simo and Taylor (1985), considering the evolution problem from a discrete incremental standpoint for a finite time step $\Delta t$ (as opposed to continuous time), the elastoplastic constitutive law reduces to giving a rule which outputs, $\sigma_{n+1}$ consistent with the yield criterion, for any given strain increment $\Delta \varepsilon_{n}=\varepsilon_{n+1}-\varepsilon_{n}$ (input) : 


$$
\sigma_{n+1}=\tilde{\sigma}\left(\varepsilon_{n}, \sigma_{n}, \bar{e}_{n}^{p}, \Delta \varepsilon_{n}\right)
$$

Here, the notation $\sigma$ symbolically denotes the action of the radial return algorithm (RRA) of Simo and Taylor (1985); $\bar{e}^{p}$ is the cumulated equivalent plastic strain:

$$
\bar{e}^{p}=\int_{0}^{2} \sqrt{\frac{2}{3}}\left\|\mathbf{d}^{p}(\tau)\right\| \mathrm{d} \tau
$$

where $\mathrm{d}^{p}$ is the plastic strain rate, with $\operatorname{tr}\left(\mathbf{d}^{p}\right)=0$. Also, the subscript $n$ in $\left(\varepsilon_{n}, \ldots\right)$ refers to time (or pseudo-time) $t_{n}$, tr is the trace of the tensor $\mathrm{d}^{p}$ and

$$
\left\|\mathbf{d}^{p}(\tau)\right\|=\left[\mathbf{d}^{p}: \mathbf{d}^{p}\right]^{1 / 2}=+\sqrt{d_{i j}^{p} d_{i j}^{p}}
$$

\section{Radial return algorithm $(R R A)$}

The RRA, which we now briefly summarize, was given in Simo and Taylor (1985), in the context of rate-independent plasticity with Von Mises pressure insensitive yield condition, and an associative flow rule. The yield condition is

$$
f(\xi, \alpha, \kappa) \equiv\|\xi\|-\sqrt{\frac{2}{3}} \kappa\left(\bar{e}^{p}\right)=0
$$

where $\xi=\mathbf{s}-\boldsymbol{\alpha}$ in terms of the deviatoric stress $\mathbf{s}=\boldsymbol{\sigma}-\frac{1}{3}(\operatorname{tr} \sigma) \mathbf{1}$ and the back stress $\alpha$. Here, 1 is the second order unit tensor. Also, $\bar{e}^{p} \rightarrow \kappa\left(\bar{e}^{p}\right)$ is the hardening rule.

A trial deviatoric stress is introduced as

$$
\xi_{n+1}^{\mathrm{T}}=\mathbf{s}_{n}+2 G \Delta \mathbf{e}_{n}-a_{n}
$$

where $\mathbf{e}=\boldsymbol{\varepsilon}-\frac{1}{3}(\operatorname{tr} \varepsilon) \mathbf{1}$ and $G$ is the shear modulus of the material.

If $f\left(\xi_{n+1}^{\mathrm{T}}, \boldsymbol{\alpha}_{n}, \kappa_{n}\right) \leqslant 0$, i.e., $\xi_{n+1}^{\mathrm{T}}$ is elastic, one has

$$
\overline{\boldsymbol{\sigma}}=K \Delta \varepsilon_{n}:(\mathbf{1} \otimes 1)+2 G \Delta \mathbf{e}_{n}+\boldsymbol{\sigma}_{n}
$$

(with $K$ the bulk modulus and $\otimes$ denoting the tensor product) i.e., the elastic constitutive equation in incremental form. On the other hand, if $f\left(\xi_{n+1}^{\mathrm{T}}, \boldsymbol{\alpha}_{n}, \kappa_{n}\right)>0, \bar{\sigma}$ is given by the following equations, which constitute the RRA :

$$
\begin{gathered}
\overline{\boldsymbol{\sigma}}=K \boldsymbol{\varepsilon}_{n+1}:(\mathbf{1} \otimes \mathbf{1})+\mathbf{s}_{n+1} \\
\mathbf{s}_{n+1}=\alpha_{n}+\sqrt{\frac{2}{3}}\left(\kappa_{n+1}+\Delta H_{n}\right) \hat{\mathbf{n}} \\
\bar{e}_{n+1}^{p}=\bar{e}_{n}^{p}+\sqrt{\frac{2}{3}}[\gamma \Delta t] \\
\hat{\mathbf{n}}=\frac{1}{\left\|\xi_{n+1}^{\mathrm{T}}\right\|} \xi_{n+1}^{\mathrm{T}}
\end{gathered}
$$

where $[\gamma \Delta t]$ solves the nonlinear consistency equation

$$
\left\|\xi_{n+1}^{\mathrm{T}}\right\|-\sqrt{\frac{2}{3}} \kappa\left(\bar{e}_{n}^{p}+\sqrt{\frac{2}{3}}[\gamma \Delta t]\right)-2 G[\gamma \Delta t]-\sqrt{\frac{2}{3}} \Delta H_{n}=0
$$

with $\Delta H_{n}=H_{n+1}-H_{n}, H_{n}=H\left(\bar{e}_{n}^{p}\right), \kappa_{n}=\kappa\left(\bar{e}_{n}^{p}\right)$. The evolution of the back-stress $\alpha_{n}$ is governed by 


$$
\boldsymbol{\alpha}_{n+1}=\boldsymbol{\alpha}_{n}+\sqrt{\frac{2}{3}} \Delta H_{n} \hat{\mathbf{n}}
$$

where $H^{\prime}\left(\bar{e}^{p}\right)$ is the plastic modulus. Here, a superposed "prime" indicates differentiation with respect to the argument.

\section{Equilibrium constraint}

In a FEM framework, the supplementary constraint provided by the need to satisfy the equilibrium equation at any time $t$ (in e.g., the virtual work form) is then used to determine which $\Delta \varepsilon$ exactly should be input in (1). The necessary condition for equilibrium at step $n+1$ is, using (1):

$$
\begin{aligned}
G(\mathbf{u}, \mathbf{v}) & \equiv \int_{\Omega} \overline{\boldsymbol{\sigma}}\left(\varepsilon_{n}, \sigma_{n}, \bar{e}_{n}^{p}, \Delta \varepsilon_{n}\right): \nabla \mathbf{v} \mathrm{d} V-\int_{\Omega} \mathbf{b}^{n+1} \cdot \mathbf{v} \mathrm{d} V-\int_{S_{T}} \mathbf{t}^{n+1} \cdot \mathbf{v} \mathrm{d} S \\
& =0 \quad(\forall \mathbf{v} \in \mathscr{V})
\end{aligned}
$$

with $2 \Delta \varepsilon_{n}=\left(\nabla+\nabla^{\mathrm{T}}\right)\left(\mathbf{u}-\mathbf{u}_{n}\right)$, and $\mathbf{v}$ is an admissible variation. Then, the displacement increment $\Delta \mathbf{u}_{n}$, such that $\mathbf{u}_{n+1}=\mathbf{u}_{n}+\Delta \mathbf{u}_{n}$ solves the nonlinear eqn (10), is sought for iteratively using a Newton method : the additive correction $\delta \mathbf{u}_{n}^{i}=\Delta \mathbf{u}_{n}^{i+1}-\Delta \mathbf{u}_{n}^{i}$ to $\Delta \mathbf{u}_{n}^{i}$ solves

$$
G\left(\mathbf{u}_{n+1}^{i}, \mathbf{v}\right)+\int_{\Omega} \frac{\partial \bar{\sigma}}{\partial \Delta \varepsilon_{n}}\left(\varepsilon_{n}, \sigma_{n}, \bar{e}_{n}^{p}, \Delta \varepsilon_{n}^{i}\right): \nabla\left(\delta \mathbf{u}_{n}^{i}\right): \nabla \mathbf{v} \mathrm{d} V=0 \quad(\forall \mathbf{v} \in \mathscr{V})
$$

with $2 \Delta \varepsilon_{n}^{i}=\left(\nabla+\nabla^{\mathrm{T}}\right) \Delta \mathbf{u}_{n}^{i}$. The local consistent tangent operator (CTO) is the fourth-order tensor $\mathbf{C}_{n+1}=\partial \bar{\sigma} / \partial \Delta \varepsilon_{n}$. It depends on the particular algorithm $\Delta \varepsilon_{n} \rightarrow \sigma_{n+1}$ chosen. The global CTO is the stiffness operator associated with the bilinear form $\delta \mathrm{u}$, $\mathbf{v} \rightarrow \int_{\Omega}\left(\mathbf{C}_{n+1}: \delta \mathbf{u}: \mathbf{v}\right) \mathrm{d} V$.

This is essentially the approach followed, in conjunction with the RRA, in Simo and Taylor (1985), where the expression of $\mathbf{C}_{n+1}$ associated with the RRA (5)-(9) is also given. It is important to note that when $\bar{\sigma}\left(\varepsilon_{n}, \sigma_{n}, \bar{e}_{n}^{p}, \Delta \varepsilon_{n}\right)$ is elastic one has

$$
\mathbf{C}_{n+1}=\mathbf{C}
$$

where $\mathbf{C}$ is the fourth-order tensor of elastic coefficients.

\section{THE GLOBAL CONSISTENT TANGENT OPERATOR USING BEM}

The virtual-work form (10) of the equilibrium equation is not suitable for boundary element-oriented formulations. In this section, it is shown how to build the global CTO when the equilibrium constraint is formulated in terms of boundary integral equations and internal representation formulas.

\section{$B E M$ formulation for elastic problems with initial strain}

We consider the initial strain approach of elastic-plastic problems. For a given initial strain distribution $\varepsilon^{p}$, the elastic constitutive law reads :

$$
\boldsymbol{\sigma}=\mathrm{C}:\left(\boldsymbol{\varepsilon}-\boldsymbol{\varepsilon}^{p}\right) \quad \varepsilon^{p}=\boldsymbol{\varepsilon}-\mathrm{C}^{-1}: \boldsymbol{\sigma}
$$

The boundary integral equation (BIE)

$$
\int_{\partial \Omega}\left[u_{i}(\mathbf{z})-u_{i}(\mathbf{x})\right] P_{i}^{k}(\mathbf{x}, \mathbf{z}) \mathrm{d} S_{z}-\int_{\partial \mathbf{\Omega}} p_{i}(\mathbf{z}) U_{i}^{k}(\mathbf{x}, \mathbf{z}) \mathrm{d} S_{z}=\int_{\Omega} U_{i, j}^{k}(\mathbf{x}, \mathbf{z}) C_{i j a b} \varepsilon_{a b}^{p}(\mathbf{z}) \mathrm{d} V_{z}
$$

where $\mathbf{x}$ is any fixed source point on the boundary $\partial \Omega$, holds for any elastic state with initial 
strain $\varepsilon^{p}$ but otherwise no domain forces; $U_{i}^{k}, P_{i}^{k}$ denote the components of the elastic singular fundamental displacement and traction, i.e., those created in the infinite space $\mathbb{R}^{3}$ by a unit point force applied at $\mathbf{x}$ along the $k$-direction, $\mathbf{p}=\sigma \cdot \mathbf{n}$ is the traction vector. The variable field point is denoted by $\mathbf{z}$ in the above equation.

The above BIE symbolically reads :

$$
[\mathbf{H}]\{\mathbf{u}\}-[\mathbf{G}]\{\mathbf{p}\}=[\mathbf{Q}]\left\{\mathbf{C}: \boldsymbol{\varepsilon}^{p}\right\}
$$

In the standiard boundary element method, the above equation is discretized and then recast as

$$
[\mathbf{A}]\{\mathbf{y}\}=\{\mathbf{f}\}+[\mathbf{Q}]\left\{\mathbf{C}: \boldsymbol{\varepsilon}^{p}\right\}
$$

where $\{\mathbf{y}\}$ collects the boundary unknowns and $\{\mathbf{f}\}$ is the contribution of known boundary variables, i.e., values prescribed by the boundary conditions.

$B E M$ representation at internal points. The displacement at any point $\mathbf{x}$ not on the boundary is then given in terms of the boundary variables $\mathbf{u}, \mathbf{p}$ and initial strain $\varepsilon^{p}$ by the representation formula:

$$
u_{k}(\mathbf{x})=\int_{\partial \Omega}\left\{p_{i}(\mathbf{z}) U_{i}^{k}(\mathbf{x}, \mathbf{z})-u_{i}(\mathbf{z}) P_{i}^{k}(\mathbf{x}, \mathbf{z})\right\} \mathrm{d} S_{z}+\int_{\Omega} U_{i, j}^{k}(\mathbf{x}, \mathbf{z}) C_{i j a b} \varepsilon_{a b}^{p}(\mathbf{z}) \mathrm{d} V_{z}
$$

Differentiation of the above formula with respect to $x_{l}$ yields the representation formula for the displacement gradient. A suitable regularization procedure which has been discussed elsewhere (Chandra and Mukherjee, 1996) is needed at this stage for the domain integral. As a result, one has

$$
\begin{aligned}
u_{k, l}(\mathbf{x})=\int_{\partial \Omega} u_{i}(\mathbf{z}) D_{i l}^{k}(\mathbf{x}, \mathbf{z}) \mathrm{d} S_{z}-\int_{\partial \Omega} p_{i}(\mathbf{z}) U_{i, l}^{k}(\mathbf{x}, \mathbf{z}) \mathrm{d} S_{z} \\
\quad-C_{i j a b} \varepsilon_{i j}^{p}(\mathbf{x}) \int_{\partial \Omega} n_{l}(\mathbf{z}) U_{a, b}^{k}(\mathbf{x}, \mathbf{z}) \mathrm{d} S_{z}-\int_{\Omega} U_{i, j l}^{k}(\mathbf{x}, \mathbf{z}) C_{i j a b}\left[\varepsilon_{a b}^{p}(\mathbf{z})-\varepsilon_{a b}^{p}(\mathbf{x})\right] \mathrm{d} V_{z}
\end{aligned}
$$

using the notation $D_{i l}^{k}=C_{i j a b} n_{j} U_{a, b l}^{k}$. The total strain at $\mathbf{x}$ is then readily obtained from the above equation. In symbolic form, one has, with $\{\varepsilon\}$ : "vector" of strains at all internal points :

$$
\begin{aligned}
\{\boldsymbol{\varepsilon}\} & =\left[\mathbf{G}^{\prime}\right]\{\mathbf{p}\}-\left[\mathbf{H}^{\prime}\right]\{\mathbf{u}\}+\left[\mathbf{Q}^{\prime}\right]\left\{\mathbf{C}: \boldsymbol{\varepsilon}^{p}\right\} \\
& =-\left[\mathbf{A}^{\prime}\right]\{\mathbf{y}\}+\left\{\mathbf{f}^{\prime}\right\}+\left[\mathbf{Q}^{\prime}\right]\left\{\mathbf{C}: \boldsymbol{\varepsilon}^{p}\right\}
\end{aligned}
$$

Substituting for $\{\mathbf{y}\}$ from (16) into the above equation, we have:

$$
\{\boldsymbol{\varepsilon}\}=\{\mathbf{n}\}+[\mathbf{S}]\left\{\mathbf{C}: \boldsymbol{\varepsilon}^{p}\right\}
$$

where

$$
\begin{aligned}
& \{\mathbf{n}\}=\left\{\mathbf{f}^{\prime}\right\}-\left[\mathbf{A}^{\prime}\right][\mathbf{A}]^{-1}\{\mathbf{f}\} \\
& {[\mathbf{S}]=\left[\mathbf{Q}^{\prime}\right]-\left[\mathbf{A}^{\prime}\right][\mathbf{A}]^{-1}[\mathbf{Q}]}
\end{aligned}
$$

Note that $\{\mathbf{n}\}$ denotes the purely elastic solution, i.e., the one obtained for the same loading but in the absence of initial strain. Then, $(13)_{2}$ is incorporated (in the form $\left.\left\{\mathbf{C}: \boldsymbol{\varepsilon}^{p}\right\}=\{\mathbf{C}: \varepsilon\}-\{\boldsymbol{\sigma}\}\right)$ into (19), giving: 


$$
\{\boldsymbol{\varepsilon}\}=\{\mathbf{n}\}+[\mathbf{S}](\{\mathbf{C}: \boldsymbol{\varepsilon}\}-\{\boldsymbol{\sigma}\})
$$

Finally, the total strain and the stress are related through :

$$
[\mathbf{S}]\{\boldsymbol{\sigma}-\mathbf{C} \boldsymbol{\varepsilon}\}-\{\mathbf{n}\}+[\mathbf{I}]\{\boldsymbol{\varepsilon}\}=\{0\}
$$

Following Telles and Carrer (1991), the above formulae for elastic problems with "initial" strain are given in accumulated form (as opposed to the rate form).

\section{Consistent tangent operator for the BEM elastic-plastic formulation}

A new implicit BEM formulation, using the CTO, is presented in this section. With $\varepsilon^{p}$ the plastic strain, we consider the evolution of the structure between time $t_{n}$ and $t_{n+1}$. One has, from (20) and using the notation $\Delta()_{n}=()_{n+1}-()_{n}$ :

$$
[\mathbf{S}]\left\{\Delta \sigma_{n}-\mathbf{C} \Delta \boldsymbol{\varepsilon}_{n}\right\}-\left\{\Delta \mathbf{n}_{n}\right\}+[\mathbf{I}]\left\{\Delta \boldsymbol{\varepsilon}_{n}\right\}=\{0\}
$$

which includes the equilibrium constraint.

On the other hand, the radial return algorithm (1) relates $\bar{\sigma}=\sigma_{n+1}=\sigma_{n}+\Delta \sigma_{n}$ to $\Delta \varepsilon_{n}$. Combining the constitutive and equilibrium equations in the form

$$
\left\{\sigma_{n}\right\}+\left\{\Delta \sigma_{n}\right\}=\{\bar{\sigma}\}
$$

(where $\Delta \sigma_{n}\left(\Delta \varepsilon_{n}, \cdot, \cdot \cdot\right)$ comes from the BEM equation while $\bar{\sigma}\left(\Delta \varepsilon_{n}, \cdot, \cdot \cdot\right)$ comes from the RRA (1)), we obtain a nonlinear equation for $\Delta \varepsilon_{n}$ of the form :

$$
\left\{G\left(\Delta \varepsilon_{n}\right)\right\} \equiv[\mathbf{S}]\left\{\bar{\sigma}\left(\varepsilon_{n}, \sigma_{n}, \bar{e}_{n}^{p}, \Delta \varepsilon_{n}\right)-\sigma_{n}-\mathbf{C} \Delta \varepsilon_{n}\right\}-\left\{\Delta \mathbf{n}_{n}\right\}+[\mathbf{I}]\left\{\Delta \varepsilon_{n}\right\}=\{0\}
$$

The Newton method can also be applied in this case, and it is readily seen that the consistent tangent operator $C_{n+1}$ appears here as well. The additive correction $\delta \varepsilon_{n}^{i}=\Delta \varepsilon_{n}^{i+1}-\Delta \varepsilon_{n}^{i}$ to $\Delta \varepsilon_{n}^{i}$ solves :

$$
\left([\mathbf{S}]\left[\mathbf{C}-\mathbf{C}_{n+1}^{i}\right]-[\mathbf{I}]\right)\left\{\delta \varepsilon_{n}^{i}\right\}=\left\{G\left(\Delta \varepsilon_{n}^{i}\right)\right\}
$$

The quantity $\left([\mathbf{S}]\left[\mathbf{C}-\mathbf{C}_{n+1}^{i}\right]-[\mathbf{I}]\right)=\left(\left[\mathbf{D}_{n+1}^{i}\right]-[\mathbf{I}]\right)$ is hereafter called the global CTO (see Kleiber et al., 1994 for the FEM version). Once the nonlinear eqn (22) is solved for $\Delta \varepsilon_{n}$, all the variables at time $t_{n+1}$ are readily computed. It is interesting to note that the Newton step (23) involves the difference [ $\left.\mathbf{C}-\mathbf{C}_{n+1}^{i}\right]$ between the elastic constitutive law and the local CTO, rather than the local CTO itself; this is entirely consistent with the fact that eqn (21) accounts for equilibrium as well as the elastic constitutive law, while, for the FEM, eqn (10) from which the Newton step (11) stems accounts for equilibrium only.

Also, it is important to note that the elastic constitutive law and the local CTO differ only at points (referred to as 'incrementally plastic') where the current strain increment has a non-zero plastic component. Hence, the Newton step (23) admits the following block decomposition :

$$
\begin{gathered}
\left(\left[\mathbf{D}_{n+1}^{i}\right]-[\mathbf{I}]\right)_{P P}\left\{\delta \varepsilon_{n}^{i}\right\}_{P}=\left\{G\left(\Delta \varepsilon_{n}^{i}\right)\right\}_{P} \\
\left\{\delta \varepsilon_{n}^{i}\right\}_{E}=\left(\left[\mathbf{D}_{n+1}^{i}\right]\right)_{E P}\left\{\delta \varepsilon_{n}^{i}\right\}_{P}-\left\{G\left(\Delta \varepsilon_{n}^{i}\right)\right\}_{E}
\end{gathered}
$$

Equations (24), (25) use the fact that

$$
\left[\mathbf{D}_{n+1}^{i}\right]_{P E}=\left[\mathbf{D}_{n+1}^{i}\right]_{E E}=[\mathbf{0}] .
$$

The subscripts $E, P$ indicate vectors and matrices restricted to the currently elastic $(E)$ or plastic $(P)$ internal nodes and collocation points. Thus, only the restriction to currently 
plastic nodes of the global CTO $\left[\mathbf{D}_{n+1}^{i}-\mathbf{I}\right]_{P P}$ need to be factored. This shows that the global CTO has to be set up and factored only at currently plastic nodes, the currently elastic part $\left\{\delta \varepsilon_{n}^{i}\right\}_{E}$ being given explicitly by (25) once (24) is solved for $\left\{\delta \varepsilon_{n}^{i}\right\}_{P}$

\section{SENSITIVITY ANALYSIS}

Now we are interested in computing the sensitivities of the mechanical variables associated with an infinitesimal perturbation of some design parameter $b$ of an unspecified nature. As a matter of fact, such sensitivities are expected to be undefined at some particular locations, e.g., on the transition interface between the current elastic and plastic zones. However, in the interest of simplicity, we elect to disregard this issue, so that sensitivities of mechanical variables are treated as continuous in the derivations that follow. The reader is referred to a very recent paper, Lee and Arora (1995), for a detailed discussion of this issue.

Since sensitivity in the present context means a comparison of two history-dependent mechanical processes, the sensitivity computation must also proceed in an incremental way, resulting in an accumulation of sensitivity increments, each of which is a solution to a linear problem. Thi adjoint structure approach (ASA), very powerful for steady-state situations, leads to regressive computations when applied to evolution problems. Applied to nonlinear, history-dependent problems, the ASA would require the storage of the whole mechanical history and of the converged tangent operators for all time steps, thus becoming rather impractical. An excellent reference for this area of research is a recent paper by Michaleris et al. (1994). In contrast, the direct differentiation approach (DDA) fits perfectly into the progressive time-stepping computation scheme. The DDA is thus preferable for nonlinear evolution problems; it is used in the present work.

\section{The DDA : principle}

Differentiation of the boundary integral eqn (16) and internal strain representation (19) with respect to $b$ for given $\varepsilon^{p}$ is a well understood process (Chandra and Mukherjee, 1996). One readily sees that the evaluation of the derivative ${ }^{*}{ }^{*}$ plays a key role. We now concentrate on this particular task.

Let us differentiate eqn (22) w.r.t. $b$, so that : (an * above a variable denotes a derivative of that variable with respect to $b$ )

$$
\begin{aligned}
{[\mathbf{S}]\left\{\frac{*}{\boldsymbol{\sigma}}\left(\varepsilon_{n}, \boldsymbol{\sigma}_{n}, \bar{e}_{n}^{p}, \Delta \varepsilon_{n}\right)-\stackrel{*}{\boldsymbol{\sigma}}_{n}-\mathbf{C} \Delta \boldsymbol{\varepsilon}_{n}\right\}+} & {[\mathbf{I}]\left\{\stackrel{*}{\Delta} \boldsymbol{\varepsilon}_{n}\right\} } \\
& +[\stackrel{*}{\mathbf{S}}]\left\{\overline{\boldsymbol{\sigma}}\left(\varepsilon_{n}, \boldsymbol{\sigma}_{n}, \bar{e}_{n}^{p}, \Delta \varepsilon_{n}\right)-\boldsymbol{\sigma}_{n}-\mathbf{C} \Delta \varepsilon_{n}\right\}-\left\{\Delta \mathbf{n}_{n}^{*}\right\}=\{0\}
\end{aligned}
$$

(assuming that $b$ is such that the elastic coefficients do not depend on it). This derivation aims at finding a governing equation for $\stackrel{*}{\Delta} \varepsilon_{n}$ in terms of known variables at times $t_{n}$ and $t_{n+1}$ and sensitivities at time $t_{n}$. Obtaining the derivatives $[\mathbf{S}]$ and $\left\{\mathbf{n}_{n}^{*}\right\}$ usually requires quite lengthy derivations and is thus by no means trivial. However, this aspect has been discussed, at length, in previous work on sensitivity analysis of elastic and elasto-plastic BEM formulations (e.g., in Chandra and Mukherjee, 1996), where the DDA has been applied on the appropriate integral eqns $(14,17$ and 18). The focus in this paper is on obtaining $\left\{\tilde{\sigma}\left(\varepsilon_{n}, \sigma_{n}, \bar{e}_{n}^{p}, \Delta \varepsilon_{n}\right)\right\}$ from the appropriate constitutive algorithm, and group the terms multiplying $\Delta \varepsilon_{n}$ and the rest separately. Of course, one expects

$$
\left\{\stackrel{*}{\sigma}\left(\varepsilon_{n}, \sigma_{n}, \bar{e}_{n}^{p}, \Delta \varepsilon_{n}\right)\right\}=\left[\frac{\partial \bar{\sigma}}{\partial \Delta \varepsilon_{n}}\right]\left\{\Delta \varepsilon_{n}\right\}+\mathscr{F}\left(\mathscr{S}_{n}, \mathscr{S}_{n}^{*}, \mathscr{S}_{n+1}\right)
$$

where $\mathscr{S}_{n}$ denotes the state at time $t_{n}$. Note that the derivative of (22) w.r.t. $b$ must be taken for the converged state $\mathscr{S}_{n+1}$, i.e., with the value of $\Delta \varepsilon_{n}$ such that $G\left(\varepsilon_{n}, \sigma_{n}, \bar{e}_{n}^{p}, \Delta \varepsilon_{n}\right)=0$. 
Sensitivity of the $R R A$

Let us first differentiate the consistency eqn (8) w.r.t. $b$ and with the converged value of $[\gamma \Delta t]$. Noting the following formulae :

$$
\begin{aligned}
& {\stackrel{\xi}{\xi_{n+1}}}^{\mathrm{T}}=\left(\mathrm{I}-\frac{1}{3} \mathbf{1} \otimes \mathbf{1}\right):\left(\boldsymbol{\sigma}_{n}+2 G \stackrel{*}{\Delta} \varepsilon_{n}\right)-{\stackrel{*}{\alpha_{n}}}^{\prime} \\
& \left\|\xi_{n+1}^{\mathrm{T}}\right\|^{*}=\hat{\mathbf{n}}: \xi_{n+1}^{*} \\
& =\hat{\mathbf{n}}:\left(\stackrel{*}{\sigma}_{n}+2 G \stackrel{*}{\Delta} \varepsilon_{n}-\stackrel{*}{\varkappa_{n}}\right) \\
& {\stackrel{*}{\kappa_{n+1}}}=\left(\left(_{\bar{e}}^{p}+\sqrt{\frac{2}{3}}[\stackrel{*}{\gamma} \Delta t]\right) \kappa_{n+1}^{\prime}\right. \\
& \stackrel{*}{\Delta} H_{n}=\Delta H_{n}^{\prime} \stackrel{*}{e}_{n}^{p}+\sqrt{\frac{2}{3}}\left[{ }^{*} \Delta t\right] H_{n+1}^{\prime}
\end{aligned}
$$

(where $\mathbf{I}$ is the fourth order symmetric unit tensor with components $\left.\frac{1}{2}\left(\delta_{i k} \delta_{j l}+\delta_{i} \delta_{j k}\right)\right)$ one gets, after grouping terms, the following scalar linear equation for $[* \Delta t]$

$$
\hat{\mathbf{n}}:\left(*_{n}+2 G \stackrel{*}{\Delta} \varepsilon_{n}-{\stackrel{*}{\boldsymbol{\alpha}_{n}}}_{n}\right)-\sqrt{\frac{2}{3}}\left(\Delta H_{n}^{\prime}+\kappa_{n+1}^{\prime}\right) \stackrel{*^{p}}{e_{n}}=\frac{2}{3}\left(\kappa_{n+1}^{\prime}+H_{n+1}^{\prime}+3 G\right)\left[{ }^{*} \Delta t\right]
$$

which readily gives

$$
2 G\left[{ }^{*} \Delta t\right]=(1-\delta) \hat{\mathbf{n}}:\left(\boldsymbol{\sigma}_{n}+2 G \stackrel{*}{\Delta} \varepsilon_{n}-\stackrel{*}{\alpha_{n}}\right)-\theta(1-\delta)\left\|\xi_{n+1}^{\mathrm{T}}\right\| \|^{*}{ }_{n}^{p}
$$

where the abbreviated notation

$$
\begin{aligned}
& \theta=\frac{1}{\left\|\xi_{n+1}^{\mathrm{T}}\right\|} \sqrt{\frac{2}{3}}\left(\Delta H_{n}^{\prime}+\kappa_{n+1}^{\prime}\right) \\
& \delta=\frac{\kappa_{n+1}^{\prime}+H_{n+1}^{\prime}}{\kappa_{n+1}^{\prime}+H_{n+1}^{\prime}+3 G}
\end{aligned}
$$

has been used.

Then, differentiation of (6) with respect to $b$ gives

$$
{\stackrel{*}{\mathbf{S}_{n+1}}}=*_{\boldsymbol{\alpha}}^{*}+\sqrt{\frac{2}{3}}\left(\kappa_{n+1}+\Delta H_{n}\right) \stackrel{*}{\hat{\mathbf{n}}}+\sqrt{\frac{2}{3}}\left(\kappa_{n+1}^{*}+\stackrel{*}{\Delta} H_{n}\right) \hat{\mathbf{n}}
$$

The sensitivity of $\hat{\mathbf{n}}$ is

$$
\begin{aligned}
\hat{\mathbf{n}} & =\frac{1}{\left\|\xi_{n+1}^{\mathrm{T}}\right\|}(\mathbf{I}-\hat{\mathbf{n}} \otimes \hat{\mathbf{n}}):{\stackrel{*}{\xi_{n+1}^{\mathrm{T}}}}^{\mathrm{T}} \\
& =\frac{1}{\left\|\xi_{n+1}^{\mathrm{T}}\right\|}\left\{\left(\mathbf{I}-\frac{1}{3} \mathbf{1} \otimes \mathbf{1}-\hat{\mathbf{n}} \otimes \hat{\mathbf{n}}\right):\left(\boldsymbol{\sigma}_{n}+2 G \stackrel{*}{\Delta \boldsymbol{\varepsilon}_{n}}\right)-(\mathbf{I}-\hat{\mathbf{n}} \otimes \hat{\mathbf{n}}): *^{*}\right\}
\end{aligned}
$$

Using eqns $(28)_{3},(28)_{4},(30)$ and (32) in eqn (31) and grouping terms appropriately, one gets

$\stackrel{\mathbf{S}}{n+1}_{n}=[(1-\beta) \mathbf{I}+(\beta-\delta) \hat{\mathbf{n}} \otimes \hat{\mathbf{n}}]: *_{n}^{*}$

$$
+\left[\beta\left(\mathbf{I}-\frac{1}{3} \mathbf{1} \otimes \mathbf{1}\right)-(\beta-\delta) \hat{\mathbf{n}} \otimes \hat{\mathbf{n}}\right]:\left({\stackrel{*}{\boldsymbol{\sigma}_{n}}}+2 G \stackrel{*}{\Delta \boldsymbol{\varepsilon}_{n}}\right)+\theta(1-\delta) \xi_{n+1}^{\mathrm{T}} \stackrel{{ }^{*}}{{ }^{p}}
$$

where the new parameter $\beta$ (as in Simo and Taylor, 1985) is : 


$$
\beta=\frac{1}{\left\|\xi_{n+1}^{\mathrm{T}}\right\|} \sqrt{\frac{2}{3}}\left(\Delta H_{n}+\kappa_{n+1}\right)
$$

Also, $\beta-\delta$ is called $\bar{\gamma}$ in Simo and Taylor (1985). Combining the above equation with the sensitivity version of (5), we obtain the sensitivity of the radial return algorithm as

$$
\begin{aligned}
& \stackrel{*}{\boldsymbol{\sigma}}=\left\{\beta\left(\mathbf{I}-\frac{1}{3} \mathbf{1} \otimes \mathbf{1}\right)-(\beta-\delta) \hat{\mathbf{n}} \otimes \hat{\mathbf{n}}\right\}::^{*} \boldsymbol{\sigma}_{n}+K(\mathbf{1} \otimes \mathbf{1})^{*} \boldsymbol{\varepsilon}_{n} \\
& +\left\{K \mathbf{1} \otimes 1+2 G \beta\left(\mathbf{I}-\frac{1}{3} 1 \otimes 1\right)-2 G(\beta-\delta) \hat{\mathbf{n}} \otimes \hat{\mathbf{n}}\right\}:{ }^{*} \varepsilon_{n} \\
& +\theta(1-\delta) \xi_{n+1}^{\mathrm{T}} \stackrel{{ }^{*}}{\stackrel{e}{n}_{n}^{p}}+\{(1-\beta) \mathbf{I}+(\beta-\delta) \hat{\mathbf{n}} \otimes \hat{\mathbf{n}}\}::_{\boldsymbol{\alpha}_{n}}^{*}
\end{aligned}
$$

Note that ${ }^{*}$ is updated using:

$$
\stackrel{*}{\alpha_{n+1}}={\stackrel{*}{a_{n}}}+\sqrt{\frac{2}{3}}\left\{\stackrel{*}{\Delta} H_{n} \hat{\mathbf{n}}+\Delta H_{n} \stackrel{*}{\hat{\mathbf{n}}}\right\}
$$

which results from a differentiation of (9) w.r.t. $b$. It is very important to note that the factor multiplying $\stackrel{*}{\Delta \varepsilon_{n}}$ in (34) is equal to the converged value of the consistent tangent operator $\mathbf{C}_{n+1}$ as given in Simo and Taylor (1985).

As mentioned at the start of this section, sensitivity equations such as (34) are not strictly valid throughout a history dependent elasto-plastic process. For example, as expected, strain sensitivities suffer jump discontinuities at the onset of plasticity. This is clear from the numerical results presented in Section 6 of this paper. Usually, however, this is not a major problem in practical optimization applications because possible local errors are not expected to significantly affect sensitivities of global functionals (usually defined over a part of a body or its boundary).

\section{Sensitivity analysis}

Finally, substitution of (34) into (27) gives a linear equation for $\left\{{ }^{*} \varepsilon_{n}\right\}$ of the form

$$
\left([\mathbf{S}]\left[\mathbf{C}-\mathbf{C}_{n+1}\right]-[\mathbf{I}]\right)\left\{\stackrel{*}{\Delta} \boldsymbol{\varepsilon}_{n}\right\}=\{\mathbf{F}\}
$$

The function $\{\mathbf{F}\}$ is completely known at this stage. It depends on the converged values of the variables at states $n$ and $n+1$ and known sensitivities-those of the stress etc. at state $n$ and the known loading sensitivity $\left\{*_{n+1}^{*}\right\}$. The matrix multiplying ${ }_{\Delta}^{*} \varepsilon_{n}$ is the converged value of the "global (or system) consistent tangent matrix" (see eqn (23)). Here it is also advisable to use the block decomposition, so that the sensitivity increment computation at currently plastic and elastic nodes is uncoupled:

$$
\begin{gathered}
\left(\left[\mathbf{D}_{n+1}^{i}\right]-[\mathbf{I})_{P P}\left\{\stackrel{*}{\Delta} \boldsymbol{\varepsilon}_{n}\right\}_{P}=\{\mathbf{F}\}_{P}\right. \\
\left\{\stackrel{*}{\Delta} \boldsymbol{\varepsilon}_{n}\right\}_{E}=\left(\left[\mathbf{D}_{n+1}^{i}\right]\right)_{E P}\left\{\stackrel{*}{\Delta} \varepsilon_{n}\right\}_{P}-\{\mathbf{F}\}_{E}
\end{gathered}
$$

As a result, the practical global CTO for both the usual mechanics and sensitivity problems is $\left(\left[\mathbf{D}_{n+1}^{i}\right]-[\mathbf{I}]\right)_{P P}$.

\section{ALGORITHM}

Let us assume that a standard discretization has been adopted, using boundary elements and domain integration cells. The latter are associated to nodal DOFs for the total strain $\varepsilon$ and stress $\sigma$, using e.g. finite element type interpolations. Note, however, that the domain discretization is restricted to the potentially plastic part of $\Omega$. Then $[\mathbf{A}]$, [S], etc. and $\{\varepsilon\}$, etc. reduce to ordinary matrices and DOF vectors, respectively. 
The following algorithm is then proposed for solving the incremental elastic-plastic problem, from initial time $t_{0}$ to final time $t_{N_{T}}$. The initial time $t_{0}$ is assumed to correspond to the first yield load.

For $0 \leqslant n \leqslant\left(N_{T}-1\right)$ :

1. Compute $\left\{\Delta \mathbf{n}_{n}\right\}$ (purely elastic internal strain).

2. Initialize $\left\{\Delta \varepsilon_{n}^{0}\right\}$ (e.g., to the elastic value).

Iterative solution of $(22)$ :

(a) $i=0$.

(b) Compute the residual $\left\{G\left(\Delta \varepsilon_{n}^{i}\right)\right\}$.

(c) Convergence test: if $\left\{G\left(\Delta \varepsilon_{n}^{i}\right)\right\} \leqslant$ EPS, GOTO 3 .

(d) $i:=i+1$.

(e) Compute the local CTOs $\mathbf{C}_{n+1}^{i}$ at all internal nodes; determine the sets of currently elastic $(E)$ and plastic $(P)$ internal nodes.

(f) Set up and factor the global CTO $\left[\mathbf{S}\left(\mathbf{C}-\mathbf{C}_{n+1}^{t}\right)-\mathbf{I}\right]_{P P}$, set up $\left[\mathbf{S}\left(\mathbf{C}-\mathbf{C}_{n+1}^{i}\right)-\mathbf{I}\right]_{E P}$.

(g) Solve (24) for $\left\{\delta \varepsilon_{n}^{i}\right\}_{P}$ and compute $\left\{\delta \varepsilon_{n}^{i}\right\}_{E}$ using (25).

(h) Update: $\left\{\Delta \varepsilon_{n}^{i}\right\}:=\left\{\Delta \varepsilon_{n}^{i}\right\}+\left\{\delta \varepsilon_{n}^{i}\right\}$.

(i) GOTO (b) (start new iteration).

3. Update: $\left\{\bar{e}^{p}\right\}_{n+1}=\left\{\bar{e}^{p}\right\}_{n}+\sqrt{\frac{2}{3}}\{[\gamma \Delta t]\},\left\{\sigma_{n+1}\right\}=\left\{\bar{\sigma}\left(\Delta \varepsilon_{n+1}^{i}\right)\right\},\left\{\boldsymbol{\varepsilon}_{n+1}\right\}=\left\{\boldsymbol{\varepsilon}_{n}\right\}+\left\{\Delta \boldsymbol{\varepsilon}_{n}\right\}$.

4. Sensitivity problem:

(a) Set up the right-hand sides $\{\mathbf{F}\}_{P},\{\mathbf{F}\}_{E}$.

(b) Solve (37) for $\left\{\stackrel{*}{\Delta} \varepsilon_{n}\right\}_{P}$ and compute $\left\{\stackrel{*}{\Delta} \varepsilon_{n}\right\}_{E}$ using (38).

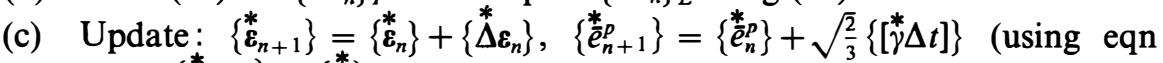
(30)), $\left\{\boldsymbol{\sigma}_{n+1}^{*}\right\}=\{\dot{\boldsymbol{\sigma}}\}$ (using eqn (34)).

Continue.

\section{EXAMPLE-A SPHERICAL CAVITY IN AN INFINITE DOMAIN}

A one-dimensional numerical example is discussed in some detail in this section. The example chosen here has closed form solutions, for both the usual mechanics problem as well as the sensitivity problem, for the case of linear work hardening materials. The purposes of this example are to illustrate the main features of the present general formulation and to check the accuracy of (some of) the numerical solutions by comparing them with the available analytical ones. Also, the efficiency of obtaining numerical solutions, as well as their robustness, are examined for this particular example.

Let $\Omega$ be the infinite three-dimensional domain exterior to a spherical cavity (radius $a$, center $O)$. A time-dependent uniform pressure $p(t)$ is applied on the cavity surface: $\mathbf{p}=-p(t) \mathbf{n}$. Usual and sensitivity elastic-plastic problems will be considered.

We denote by $(r, \theta, \phi)$ a system of spherical coordinates centered at $O$, so that $\Omega=[a,+\infty[\times \mathscr{S}$ and $\partial \Omega=\{r=a\} \times \mathscr{S}, \mathscr{S}$ being the unit sphere associated with the spherical angles $(\theta, \phi)$. Due to the spherical symmetry, the example is essentially onedimensional: the only nonzero mechanical variables, namely $u_{r}, \varepsilon_{r r}, \varepsilon_{\theta \theta}=\varepsilon_{\phi \phi}, \sigma_{r r}, \sigma_{\theta \theta}=\theta_{\phi \phi}$, depend only on the radial coordinate $r$. The strain and stress deviatoric tensors $\mathbf{e}$ and $\mathbf{s}$ then have the form

$$
\mathbf{e}=e \mathbf{a} \quad \mathbf{s}=s \mathbf{a} \quad \text { with } \quad \mathbf{a}=\operatorname{Diag}\left[\frac{2}{3},-\frac{1}{3},-\frac{1}{3}\right]
$$

in terms of the scalar deviatoric strain $e=\varepsilon_{r r}-\varepsilon_{\theta \theta}$ and stress $s=\sigma_{r r}-\sigma_{\theta \theta}$. The material has isotropic elastic properties and obeys a von Mises yield criterion with nonlinear isotropic hardening $\left(H^{\prime}\left(\bar{e}^{p}\right)=0\right)$, which, since $\|\mathbf{a}\|=\sqrt{\frac{2}{3}}$, reduces to (see eqn 2):

$$
|s|-\kappa\left(\bar{e}^{p}\right) \leqslant 0, \quad \kappa=2 G\left(k_{0}+k_{1}\left(\bar{e}^{p}\right)^{m}\right)
$$

where $k_{0}, k_{1}$ and $m$ are material parameters. 
For this problem, first yielding occurs at $r=a$ at the yield pressure $p_{Y}=(4 G / 3) k_{0}$. A nondimensional load parameter $\lambda$ is defined as

$$
p(t)=p_{Y}(1+\lambda(t))=\frac{4 G}{3} k_{0}(1+\lambda(t))
$$

The exact solution of the problem, for the linear hardening case $(m=1)$ is given in the Appendix for completeness.

\section{Integral equations and representations}

It is instructive to observe the one-dimensional counterparts of the key equations that are given in general form in the earlier sections of this paper. Some of these are given below and the rest are given in the Appendix.

Let $\mathbf{x}(r, \theta=0, \phi=0)$, with $r \geqslant a$, denote a fixed point of $\Omega$. Due to the spherical symmetry of the problem and assuming the 'initial' strain $\varepsilon^{p}$ to be purely deviatoric, the integral representation (17) reduces to

$$
\begin{aligned}
u_{r}(r)+u_{\rho}(a) & \int_{\mathscr{S}} P_{\rho}^{r}(\mathbf{x}, \mathbf{z}(a, \theta, \phi)) a^{2} \mathrm{~d} \omega-\sigma_{\rho \rho}(a) \int_{\mathscr{S}} U_{\rho}^{r}(\mathbf{x}, \mathbf{z}(a, \theta, \phi)) a^{2} \mathrm{~d} \omega \\
& =2 G \int_{a}^{+\infty}\left[\varepsilon_{\rho \rho}^{p}-\varepsilon_{\theta \theta}^{p}\right](\rho)\left\{\int_{\mathscr{S}}\left[\frac{2}{3} E_{\rho \rho}^{r}-\frac{1}{3} E_{\theta \theta}-\frac{1}{3} E_{\phi \phi}\right](\mathbf{x}, \mathbf{z}(\rho, \theta, \phi)) \mathrm{d} \omega\right\} \rho^{2} \mathrm{~d} \rho
\end{aligned}
$$

with $\mathrm{d} \omega=\sin \phi \mathrm{d} \theta \mathrm{d} \phi$. Here $U^{r}(\mathbf{x}, \mathbf{z}), P^{r}(\mathbf{x}, z)$ are the Kelvin fundamental solutions for isotropic elasticity, with the point force applied in the fixed $r$-direction at $\mathbf{x}$, and $E^{r}(\mathbf{x}, \mathbf{z})$ is the strain tensor associated with the displacement $U^{r}$. Integrations w.r.t. $\theta, \phi$ can be carried out analytically, and as a result the above equation becomes

$$
u_{r}(r)=\zeta \frac{a^{2}}{r^{2}} u_{r}(a)-\frac{1-\zeta}{2} \frac{a^{3}}{r^{2}} \frac{\sigma_{r r}(a)}{2 G}-(1-\zeta) r \int_{r}^{+\infty} e^{p}(\rho) \frac{\mathrm{d} \rho}{\rho}
$$

where $\zeta$ is defined in the Appendix.

Using the boundary condition $\sigma_{r r}(a)=-p$, one gets the explicit form

$$
u_{r}(a)=a\left\{\frac{k_{0}}{3}(1+\lambda)-\int_{a}^{+\infty} e^{p}(\rho) \frac{\mathrm{d} \rho}{\rho}\right\}
$$

The one-dimensional counterparts of eqns (19) and (20) are given in the Appendix.

\section{Local and global consistent tangent operators}

One of the primary contributions of this paper is the proposal to combine the BEM eqn (21) with the RRA to get eqn (22), and then to set up a Newton scheme to solve eqn (22). We feel that it is very important to illustrate this key procedure, in some detail, for the spherical cavity problem at hand. This is done below.

Let us consider a loading process of the form (40), so that each load step is defined by a given increment $\Delta \lambda_{n}, n \geqslant 0$. Equation (A.5), taken at the beginning and the end of a load step, gives the following relation, which has the structure of eqn (21):

$$
S\left(\Delta s_{n}-2 G \Delta e_{n}\right)-\Delta n_{n}+\Delta e_{n}(r)=0
$$

with 


$$
\begin{gathered}
S(v)=3 \zeta \frac{a^{3}}{r^{3}} \int_{a}^{+\infty} \frac{v(\rho)}{2 G} \frac{\mathrm{d} \rho}{\rho}+(1-\zeta) \frac{v(r)}{2 G} \\
\Delta n_{n}=\frac{a^{3}}{r^{3}} \Delta \lambda_{n}
\end{gathered}
$$

On the other hand, the value of $\Delta \mathrm{s}_{n}$ output by the RRA for a given input $\Delta \mathrm{e}_{n}$, for the particular situation at hand, is

$$
\overline{\Delta s}_{n}\left(e_{n}, s_{n}, \bar{e}_{n}^{p}, \Delta e_{n}\right)=2 G\left(\Delta e_{n}-\chi_{n+1} \varepsilon_{n+1}[\gamma \Delta t]\right), \quad \varepsilon_{n+1}=\operatorname{sign}\left(s_{n}+2 G \Delta e_{n}\right)
$$

where the coefficient $\chi_{n+1}$ is equal to unity if the trial stress $s_{n}+2 G \Delta \mathrm{e}_{n}$ violates the von Mises criterion (39) and to zero otherwise, and $[\gamma \Delta t]$ solves the consistency eqn (8), which reads here:

$$
\begin{gathered}
\sqrt{\frac{2}{3}} \varepsilon_{n+1}\left(s_{n}+2 G \Delta e_{n}\right)-\sqrt{\frac{2}{3}} \kappa_{n+1}-2 G[\gamma \Delta t]=0 \\
\kappa_{n+1}=2 G\left\{k_{0}+k_{1}\left(\bar{e}_{n}^{p}+\sqrt{\frac{2}{3}}[\gamma \Delta t]\right)^{m}\right\}
\end{gathered}
$$

Then, the local CTO is readily obtained as

$$
\frac{\partial \overline{\Delta s}_{n}}{\partial \Delta e_{n}}=2 G\left(1-\chi_{n+1} d_{n+1}\right) \quad \text { with } \quad d_{n+1}=\frac{3 G}{3 G+\kappa_{n+1}^{\prime}}
$$

For the special case $m=1$, the consistency equation is linear and (46) holds with $\kappa_{n+1}^{\prime}=2 G k_{1}$.

Accounting for the action (45) of the RRA, eqn (44) becomes

$$
G\left(\Delta e_{n} ; r\right) \equiv S\left(\overline{\Delta s}_{n}\left(e_{n}, s_{n}, \bar{e}_{n}^{p}, \Delta e_{n}\right)-2 G \Delta e_{n}\right)-\Delta n_{n}+\Delta e_{n}(r)=0
$$

and the unknown $\Delta e_{n}$ is to be found using Newton iterations, i.e., by solving the following linear equation on the additive correction $\delta e_{n}^{i}$ until convergence of the residual $G$ :

$$
\begin{gathered}
D_{n+1}^{i}\left(\delta e_{n}^{i}\right)-\delta e_{n}^{i}(r)=G\left(\Delta e_{n}^{i} ; r\right) \\
D_{n+1}^{i}(v)=(1-\zeta)\left[\chi_{n+1}^{i} d_{n+1}^{i} v\right](r)+3 \zeta \frac{a^{3}}{r^{3}} \int_{a}^{+\infty}\left[\chi_{n+1}^{i} d_{n+1}^{i} v\right](\rho) \frac{\mathrm{d} \rho}{\rho}
\end{gathered}
$$

Note that the integration range in the integral operators $S, D$ is in practice limited to those points $\rho \in\left[a,+\infty\right.$ [ at which the current $s_{n}+\overline{\Delta s_{n}^{i}}$ is plastic, i.e., $\chi_{n+1}^{i}(\rho)=1$.

\section{Governing equations for the sensitivity problem}

Sensitivities with respect to material parameters have been considered in this paper. Numerical results are given below for $b=k_{1}$ or $b=m$.

Straightforward calculations in the same spirit as in Section 4 lead to the following formulae:

$$
\begin{aligned}
& {[\stackrel{*}{\gamma \Delta t}]=\frac{3}{6 G+2 \kappa_{n+1}^{\prime}} \sqrt{\frac{2}{3}}\left\{\varepsilon_{n+1}\left[\stackrel{*}{s}_{n}+2 G \stackrel{*}{\Delta} e_{n}\right]-\left(\kappa_{, b}\right)_{n+1}-\kappa_{n+1}^{\prime}{ }^{*}{ }^{p}{ }_{n}^{p}\right\}} \\
& \stackrel{*}{\Delta} s_{n}=2 G\left(1-\chi_{n+1} d_{n+1}\right) \stackrel{*}{\Delta} e_{n}+\chi_{n+1} d_{n+1}\left\{\varepsilon_{n+1}\left[\left(\kappa_{, b}\right)_{n+1}+\kappa_{n+1}^{\prime} \stackrel{{ }^{*}}{\stackrel{*}{e}_{n}^{p}}\right]-{\stackrel{*}{S_{n}}}_{n}\right\}
\end{aligned}
$$

The strain increment sensitivity $\stackrel{*}{\Delta e_{n}}$ then solves the linear problem 


$$
D_{n+1}\left(\stackrel{*}{\Delta} e_{n}\right)-\stackrel{*}{\Delta} e_{n}(r)=S(v), \quad v=\chi_{n+1} d_{n+1}\left\{\varepsilon_{n+1}\left[\left(\kappa_{, b}\right)_{n+1}+\kappa_{n+1}^{\prime} \stackrel{*}{e}_{n}^{p}\right]-{ }^{*} S_{n}\right\}
$$

The governing operator is the converged global CTO, which has already been set up and factored during the elastoplastic state solution step.

\section{Internal discretization}

The numerical treatment needs a discretization of the potential plastic zone $[a, c](c$ being arbitrarily chosen by the user) into integration cells. Here, a constant size $\Delta r=(c-a) / N$ is used, together with a piecewise linear and continuous interpolation for the scalar deviatoric strain $e$ and stress $s$. Substitution of this interpolation into eqns (44), (48) collocated at the radii $r=r_{k}=a+k \Delta r$ leads to the discretized nonlinear equation and associated Newton step.

Since the operator $\left[D_{n+1}^{i}\right]$ yields nonzero results only when applied to a currently plastic $\left\{\delta e_{n+1}^{i}\right\}$, it is readily seen that the Newton step (48) takes the following matrix form, where the currently elastic and plastic nodal values are uncoupled:

$$
\begin{gathered}
{\left[D_{n+1}^{i}-I\right]_{P P}\left\{\delta e_{n}^{i}\right\}_{P}=[S]_{P P}\left\{\bar{\Delta} s_{n}^{i}-2 G \Delta e_{n}^{i}\right\}-\left\{\Delta n_{n}\right\}_{P}+\left\{\Delta e_{n}^{i}\right\}_{P}} \\
\left\{\delta e_{n}^{i}\right\}_{E}=\left[D_{n+1}^{i}\right]_{E P}\left\{\delta e_{n}^{i}\right\}_{P}-[S]_{E P}\left\{\bar{\Delta} s_{n}^{i}-2 G \Delta e_{n}^{i}\right\}_{P}-\left\{\Delta n_{n}\right\}_{E}-\left\{\Delta e_{n}^{i}\right\}_{E}
\end{gathered}
$$

with

$$
\begin{aligned}
& \left\{\delta e_{n}^{i}\right\}=\left\{\delta e_{n}^{i}\left(r_{k}\right)\right\}_{0 \leqslant k \leqslant N}, \quad\left\{\Delta e_{n}\right\}=\left\{\Delta e_{n}\left(r_{k}\right)\right\}_{0 \leqslant k \leqslant N} \\
& \left\{\Delta s_{n}\right\}=\left\{\Delta s_{n}\left(r_{k}\right)\right\}_{0 \leqslant k \leqslant N}, \quad\left\{\Delta n_{n}\right\}=\left\{\frac{a^{3}}{r_{k}^{3}} \Delta \lambda_{n}\right\}_{0 \leqslant k \leqslant N}
\end{aligned}
$$

\section{Numerical results: linear isotropic hardening case}

In this example (referred to as 'example 1'), the following material parameter values were used : $m=1$ (linear hardening), $k_{0}=k_{1}=0.001$ ( $G, a$ were used as scaling parameters in the implementation, so that their precise values have no influence on the results). A potential plastic radius $c / a=4$ has been used.

First, an increasing loading process, with $\lambda_{\text {final }}=3$ (i.e., so that $p_{\text {final }}=4 p_{Y}$ ), is considered. Figure 1 shows the computed final values of the total strain $e$ and accumulated plastic strain $\bar{e}^{p}$, compared against the analytical solution, while Figs 2 and 3 depict the final sensitivity with respect to the hardening coefficient $k_{1}$ of $e$ and $\bar{e}^{p}$, respectively. Two grid sizes were considered: $\Delta r / a=0.1$ (ccarse mesh, $N=30$ ) and $\Delta r / a=0.05$ ( finer mesh, $N=60$ ). Both the elastoplastic state and its $k_{1}$-sensitivities compare well with the analytical solutions. The finer mesh gives very good sensitivity values, and catches accurately the sensitivity jump at the plastic-elastic transition radius $r=b \approx 2.70 a$. Figure 4 , which shows the $k_{1}$-sensitivities for $2.6 a \leqslant r \leqslant 2.9 a$ (zoom arouncl $r=b)$ and using two fine meshes $(\Delta r / a=0.05$ and $\Delta r / a=0.01)$, highlights the convergence towards the analytical solution; the computed values for the finest mesh reproduce accurately the expected sensitivity jumps (see Fig. 4).

Similar results have been obtained with the same data and domain grids as above but for a smaller final load $\lambda_{\text {final }}=1\left(p_{\text {final }}=2 p_{Y}\right)$. The accuracy for both state and sensitivity variables improve for $\lambda=1$ compared to $\lambda=3$. The results for both cases are acceptable.

It should be emphasized that in both cases the numerical results obtained proved to be virtually independent on the number of load steps used. Indeed, all previous results as shown were computed using just one single load increment: $\Delta \lambda=\lambda_{\text {final }}$. This fact has been consistently cobserved in all numerical tests performed on this problem. For example, another run with $\lambda_{\text {final }}=5$ and $\Delta r / a=0.05$ also gave almost identical results; 1 load step reached convergence after 6 Newton iterations on the equilibrium eqn (52), while 5 and 10 equal load increments consumed respectively 14 and 22 Newton iterations.

On the other hand, for a fixed grid size $\Delta r / a$, the final accuracy deteriorates as the final load increases; this trend has also been observed consistently in all tests performed. 


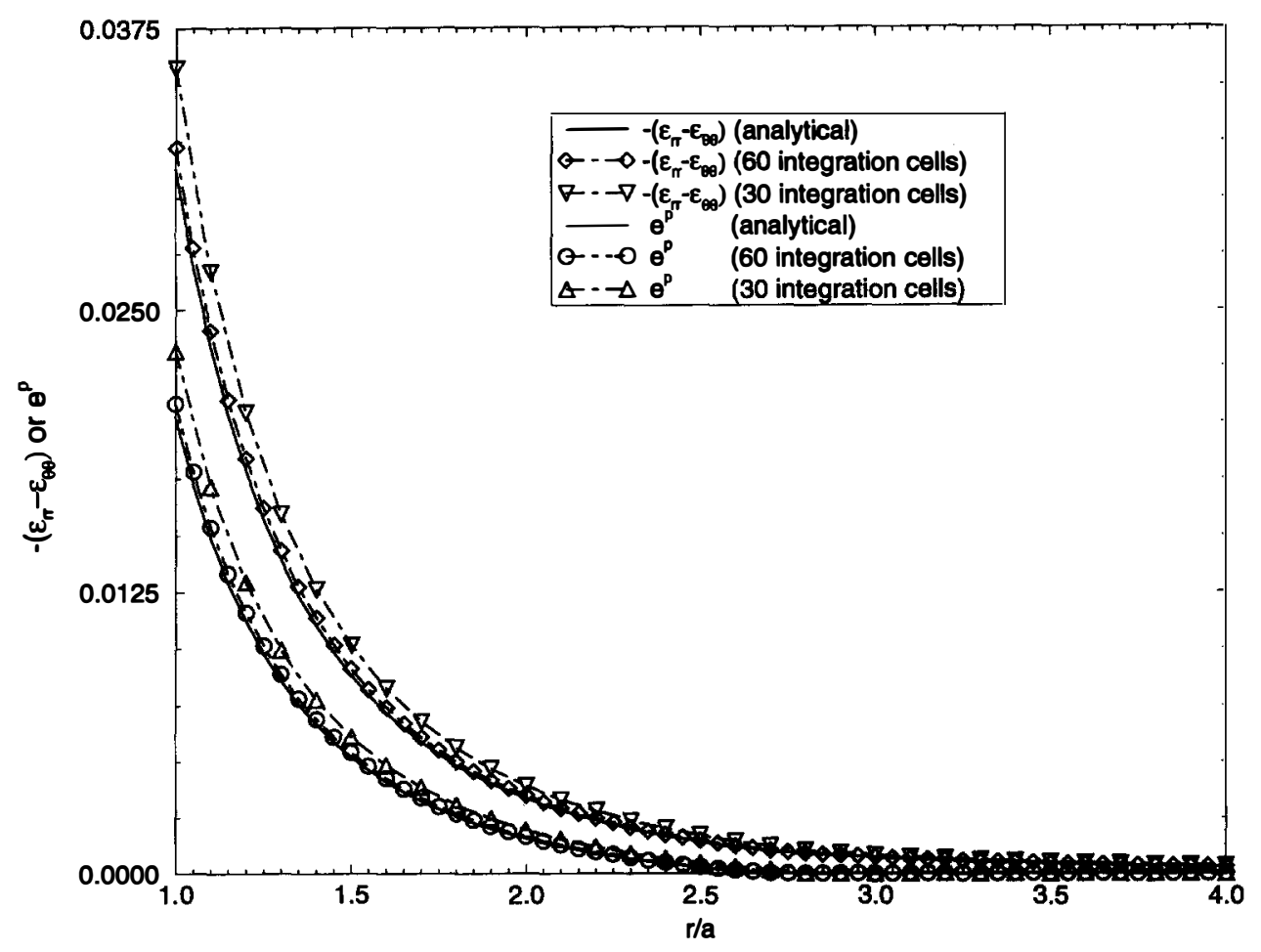

Fig. 1. Example 1: total $(e)$ and accumulated plastic $\left(\bar{e}^{p}\right)$ strains, using 30 or 60 integration cells1 load step, $\lambda_{\text {final }}=3$.

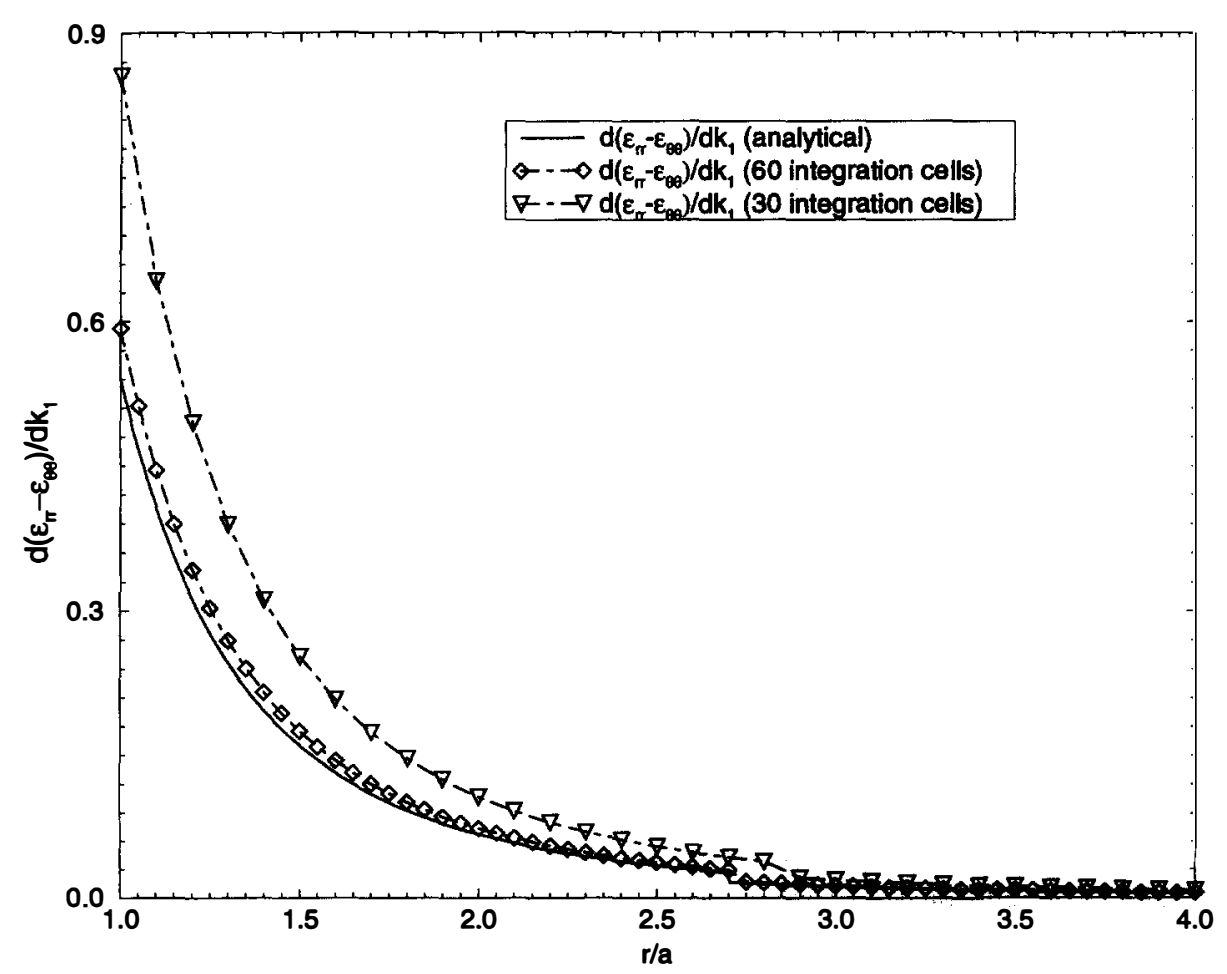

Fig. 2. Example $1: k_{1}$-sensitivity of total strain (e), using 30 or 60 integration cells-1 load step, $\lambda_{\text {finat }}=3$.

Tables 1 and 2 show clearly the convergence of the computed state and sensitivity variables towards the analytical solution with decreasing grid size $\Delta r / a$, for $\lambda_{\text {final }}=1$ and $\lambda_{\text {final }}=3$ respectively. Consistent with previously mentioned observations, the error levels 


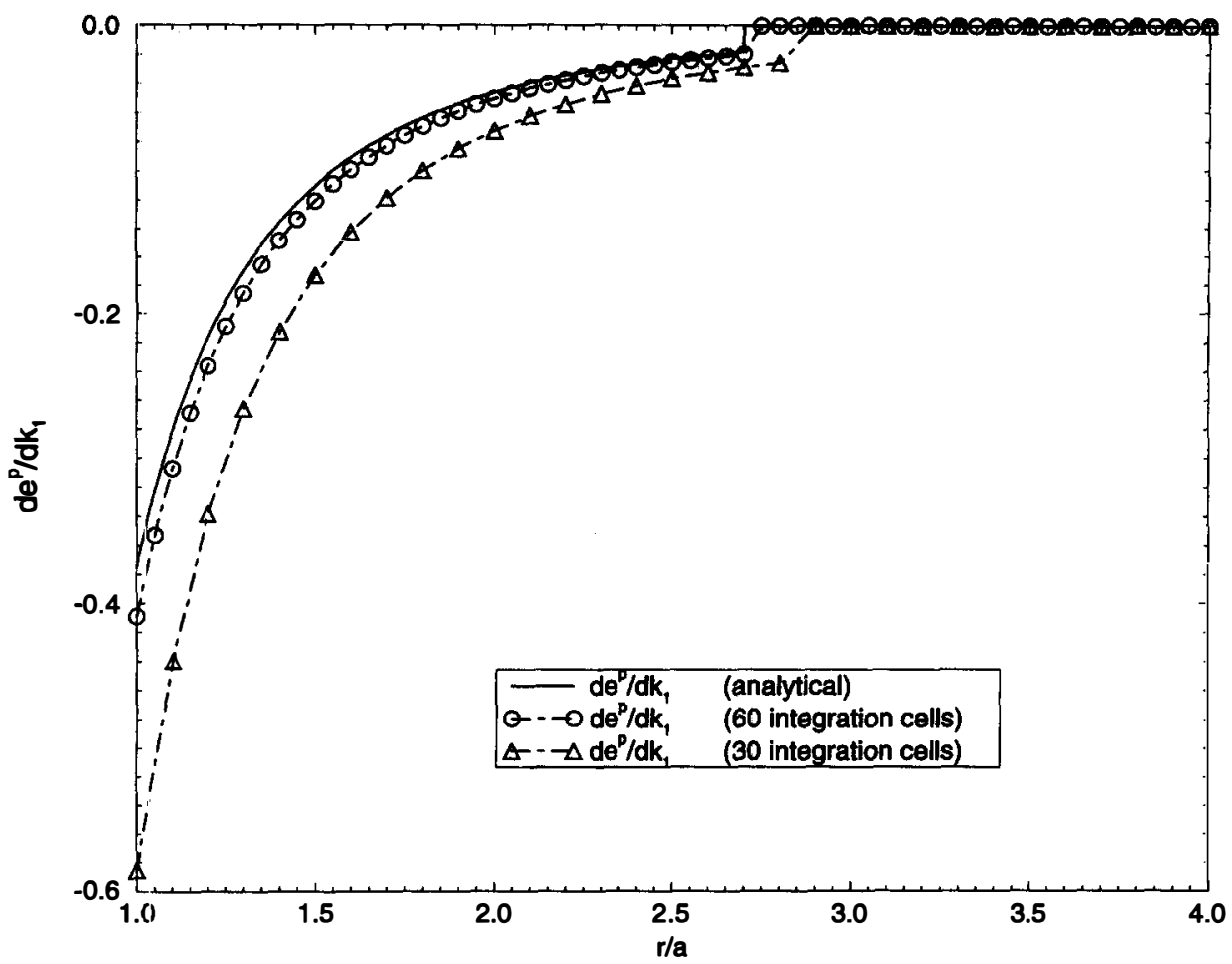

Fig. 3. Example $1: k_{1}$-sensitivity of accumulated plastic strain $\left(\bar{e}^{p}\right)$, using 30 or 60 integration cells1 load step, $\lambda_{\text {final }}=3$.

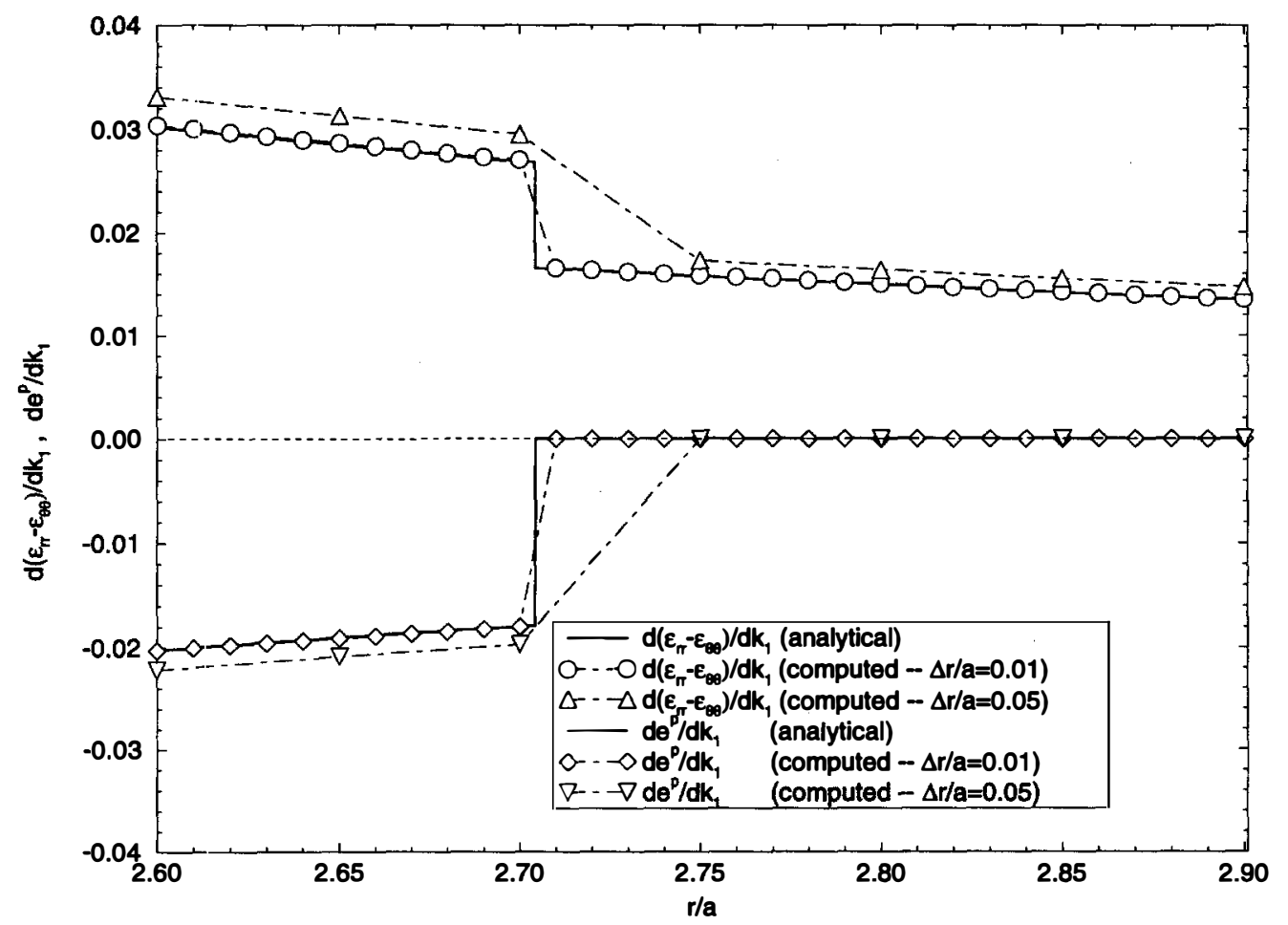

Fig. 4. Example 1: $k_{1}$-sensitivity of total $(e)$ and accumulated plastic $\left(\bar{e}^{p}\right)$ strains : zoom around the plastic/elastic transition radius -1 load step, $\lambda_{\text {fnal }}=3$.

with a fixed mesh size are higher for $\lambda_{\text {final }}=3$ than for $\lambda_{\text {final }}=1$; however the convergence is clear in both cases. Here also, one single load increment was used. The tables also show the total number of Newton iterations (from 2 to 5 ) needed for convergence and report the bracketing of the elastic-plastic radius $b$. 
Table 1. Elastoplastic and sensitivity results : evolution of $t^{2}$ relative error with decreasing integration cell size -1 load step, $\lambda_{\text {final }}=1$

\begin{tabular}{lllll}
\hline$\Delta r / a$ & 0.1 & 0.05 & 0.01 & 0.005 \\
\hline Total number of iterations & 3 & 2 & 3 & 3 \\
$b / a$ bracketing $\left(b_{\text {exact }}=1.39525 a\right)$ & {$[1.4,1.5]$} & {$[1.35,1.4]$} & {$[1.39,1.4]$} & {$[1.395,1.4]$} \\
$L^{2}$ relative error $(e)$ & $7.64 \mathrm{E}-03$ & $1.60 \mathrm{E}-03$ & $3.76 \mathrm{E}-05$ & $6.66 \mathrm{E}-7$ \\
$L^{2}$ relative error $\left(e^{0}\right)$ & $1.19 \mathrm{E}-2$ & $2.55 \mathrm{E}-03$ & $6.21 \mathrm{E}-05$ & $1.11 \mathrm{E}-6$ \\
$L^{2}$ relative error $\left(\mathrm{d} e / \mathrm{d} k_{1}\right)$ & $7.5 \mathrm{E}-02$ & $6.54 \mathrm{E}-03$ & $5.95 \mathrm{E}-05$ & $2.88 \mathrm{E}-5$ \\
$L^{2}$ relative error $\left(\mathrm{d} \tilde{e}^{p} / \mathrm{d} k_{1}\right)$ & $5.91 \mathrm{E}-02$ & $4.21 \mathrm{E}-03$ & $5.95 \mathrm{E}-05$ & $2.15 \mathrm{E}-5$ \\
\hline
\end{tabular}

Table 2. Elastoplastic and sensitivity results: evolution of $L^{2}$ relative error with decreasing integration cell size--1 load step, $\lambda_{\text {final }}=3$

\begin{tabular}{lllll}
\hline$\Delta r / a$ & 0.1 & 0.05 & 0.01 & 0.005 \\
\hline Total number of iterations & 4 & 4 & 5 & 5 \\
$b / a$ bracketing $\left(b_{\text {exact }}=2.70296 a\right)$ & {$[2.8,2.9]$} & {$[2.7,2.75]$} & {$[2.7,2.71]$} & {$[2.7,2.705]$} \\
$L^{2}$ relative error $(e)$ & $3.34 \mathrm{E}-02$ & $5.35 \mathrm{E}-03$ & $9.31 \mathrm{E}-05$ & $1.64 \mathrm{E}-05$ \\
$L^{2}$ relative error $\left(\bar{e}^{p}\right)$ & $3.55 \mathrm{E}-02$ & $5.70 \mathrm{E}-03$ & $9.96 \mathrm{E}-05$ & $1.76 \mathrm{E}-05$ \\
$L^{2}$ relative error $\left(\mathrm{d} e / \mathrm{d} k_{1}\right)$ & $1.32 \mathrm{E}-01$ & $1.59 \mathrm{E}-02$ & $3.66 \mathrm{E}-04$ & $9.08 \mathrm{E}-06$ \\
$L^{2}$ relative error $\left(\mathrm{d} \bar{e}^{p} / \mathrm{d} k_{1}\right)$ & $1.29 \mathrm{E}-01$ & $1.56 \mathrm{E}-02$ & $3.57 \mathrm{E}-04$ & $8.77 \mathrm{E}-06$ \\
\hline
\end{tabular}

\section{Numerical results : nonlinear isotropic hardening case}

This example (referred to as 'example 2') used the values $k_{0}=k_{1}=0.001$ together with various values of the hardening exponent $m$. No exact solution is available for this example. Except when indicated otherwise, the results were computed using one load increment.

Figure 5 shows the computed final values of the total strain $e$ and accumulated plastic strain $\bar{e}^{p}$ for several grid sizes (final load: $\lambda_{\text {final }}=3$, potential plastic zone boundary: $c=3 a$ ); the hardening exponent value is $m=0.1$. The results for a very fine mesh (500 cells), labelled "converged", are included so as to show the convergence behaviour with decreasing $\Delta r / a$. Actual convergence clearly occurs, and the nodal values of $e, \bar{e}^{p}$ obtained

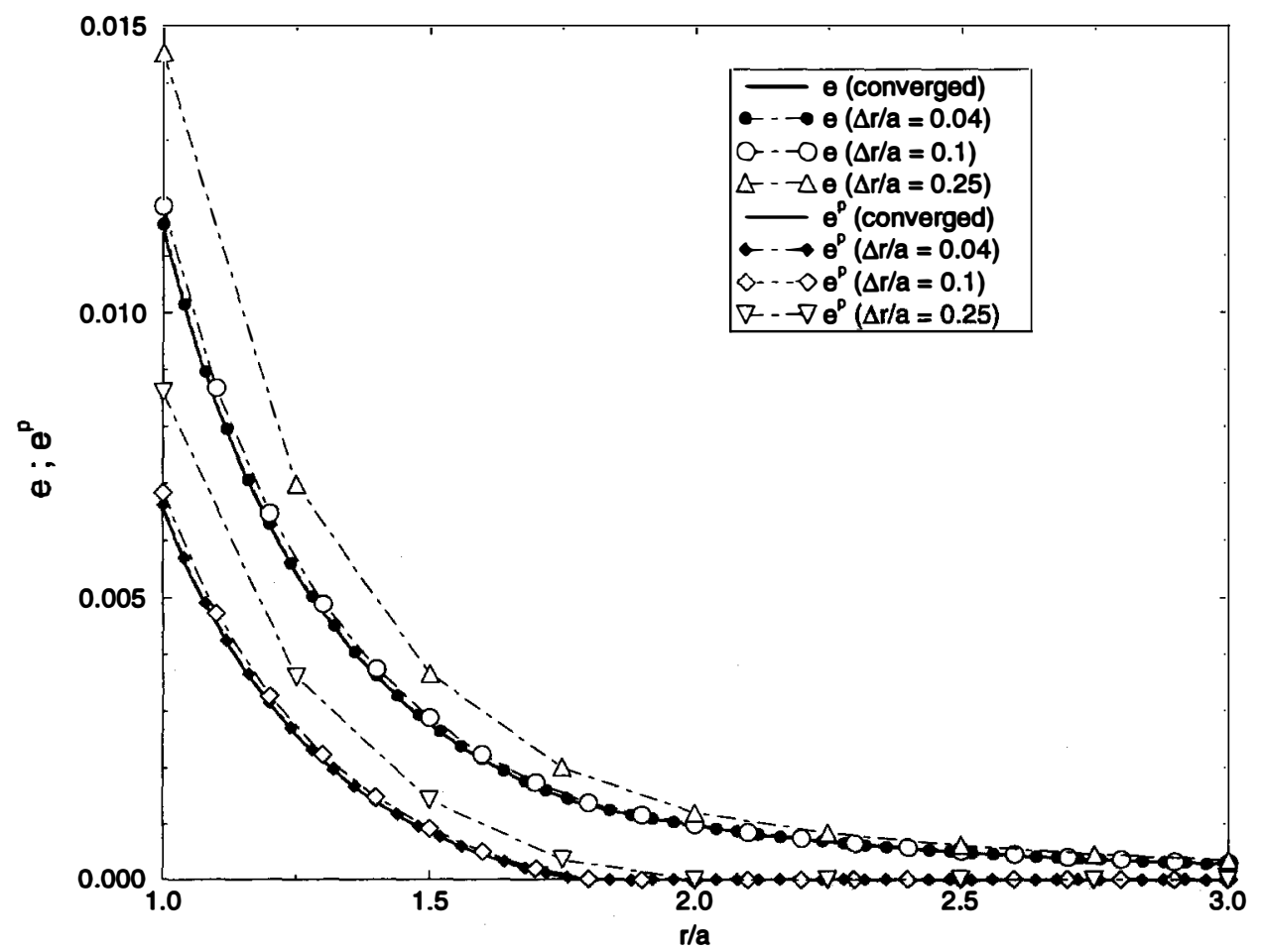

Fig. 5. Example 2: convergence of total $(e)$ and accumulated plastic $\left(\bar{e}^{p}\right)$ strains-1 load step, $\lambda_{\text {final }}=3, m=0.1$. 


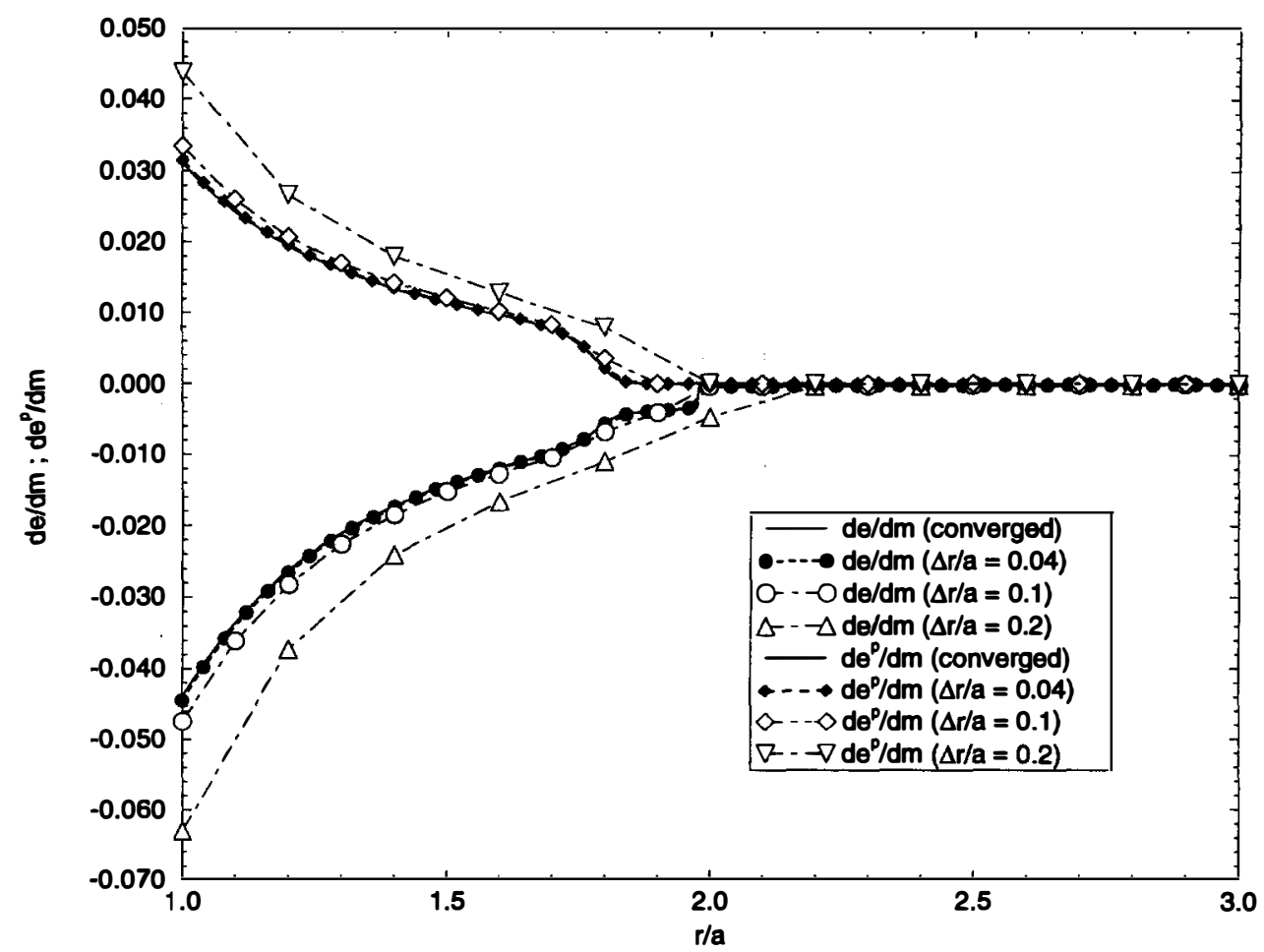

Fig. 6. Example 2: convergence of $m$-sensitivities of total $(e)$ and accumulated plastic $\left(e^{p}\right)$ strains-1 load step, $\lambda_{\text {final }}=3, m=0.1$.

using 10 radial cells $(\Delta r / a=0.1)$ are quite close to the converged solution, despite the relatively large value of the load increment $\left(\Delta \lambda=\lambda_{\text {fral }}=3\right)$. Similar observations are true for the $m$-sensitivity of $e$ and $\bar{e}^{p}$, shown in Fig. 6, also for $m=0.1$. Figure 7 shows the computed $m$-sensitivities of $\tilde{e}^{p}$ for several values of the hardening exponent.

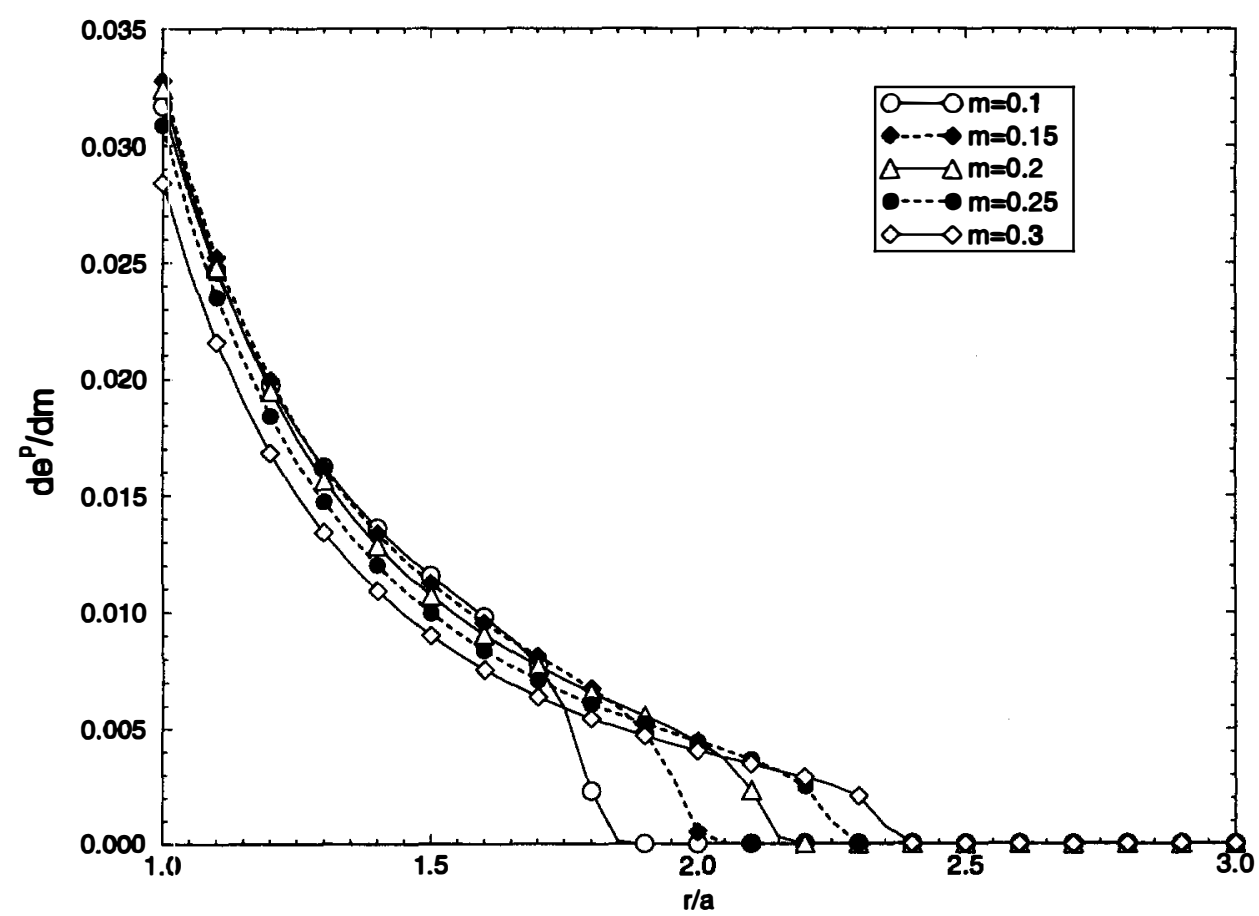

Fig. 7. Example 2: $m$-sensitivities of accumulated plastic strain $\ddot{e}^{p}$ for various values of exponent $m$-total load increment : $\lambda_{\text {final }}=3 ; \Delta r / a=0.05$. 


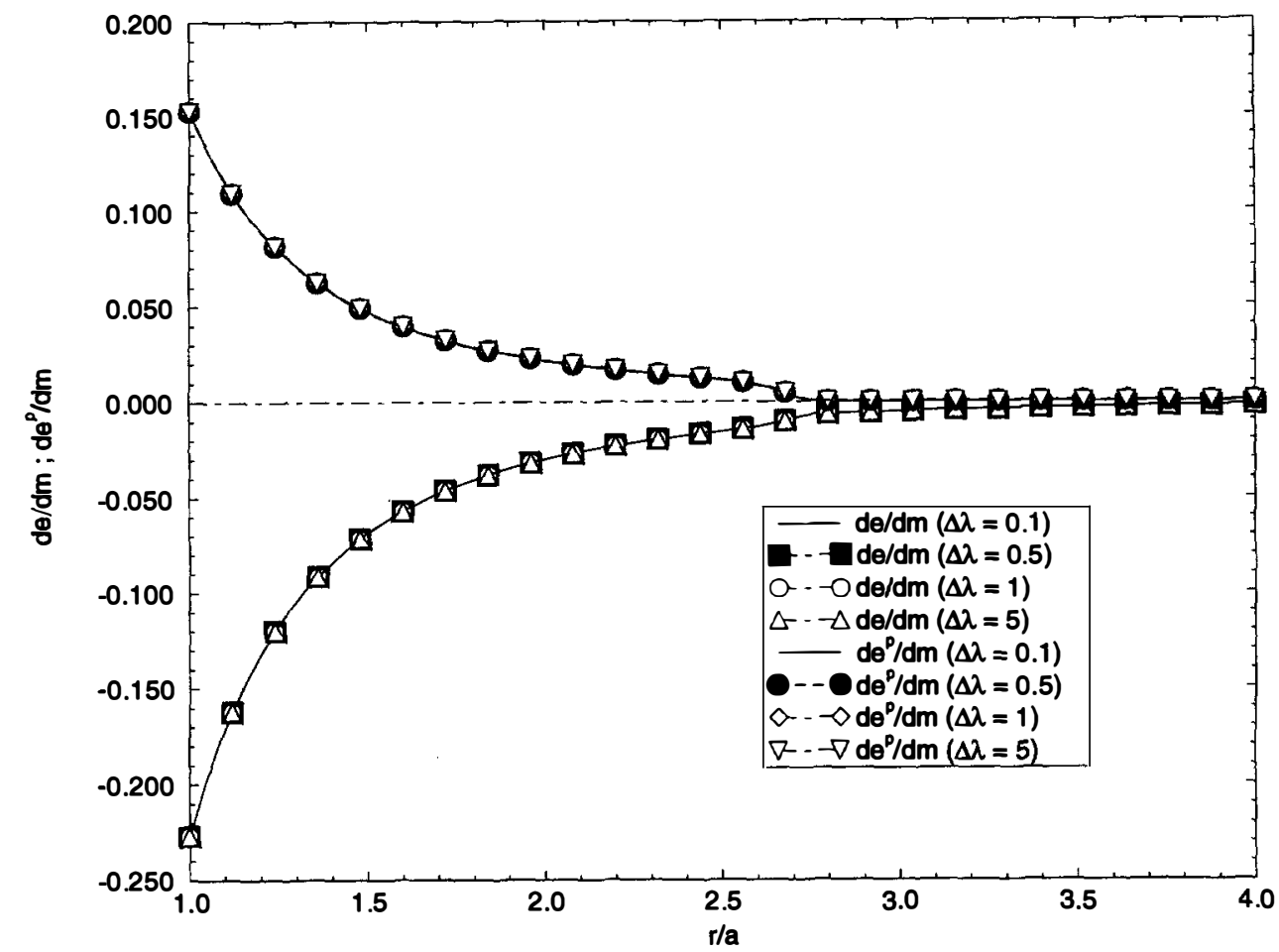

Fig. 8. Example 2 : independence of $m$-sensitivities of total $(e)$ and accumulated plastic $\left(\bar{e}^{p}\right)$ strains with respect to load step--total load increment : $\lambda_{\text {final }}=5 ; \Delta r / a=0.04$.

Similar results have been computed for, respectively, the state variables and their $m$ sensitivities, in the same spirit as above, for a final load $\lambda_{\text {final }}=1$, a potential plastic zone boundary: $c=1.5 a$ and $m=0.1$; the same grid sizes as in Figs 5 and 6 were used. One observes again, for a fixed grid size, some improvement in accuracy for $\lambda_{\text {final }}=1$ compared to $\lambda_{\text {final }}=3$. The reader is referred to, for example, chapter 11 of Haslinger and Neittaanmaki (1988) for a discussion of finite element grid optimization based on optimization techniques.

As expected, the supplementary CPU time consumed by the sensitivity calculation is very small compared with that necessary for the elastoplastic state calculation. This comment is true for all sensitivity calculations presented in this paper. For example, with $N=600$ and 50 load steps, CPU times of about 153 and 147 seconds were observed, depending on whether the sensitivities were evaluated or not.

Finally, it is important to point out that even in the presence of nonlinear hardening the results for a given final load and grid size appear to be virtually independent of the number of load steps used. This is apparent in Fig. 8, in which the computed $m$-sensitivities are shown for a large final load $\left(\lambda_{\text {final }}=5\right)$ and a fixed grid size $(\Delta r / a=0.04)$ : the final results obtained using $1,5,10$ or 50 load steps (and respectively 8, 24, 40 and 147 Newton iterations) are almost identical.

All computations were carried out on an APOLLO/HP 735 workstation.

\section{CONCLUSIONS}

Within the context of the finite element method, the value of the consistent tangent operator (CTO) for obtaining implicit solutions of elastoplastic problems has been demonstrated by, among others, Simo and Taylor (1985). It has been shown that use of other operators, such as the continuum tangent, leads to loss of quadratic convergence of the associated Newton method that is used for obtaining stepwise iterative solutions of the nonlinear problem. The associated sensitivity problem is stepwise linear. In this case, use of the CTO leads to a stiffness matrix which is the converged value of the global CTO for that load step (see, for example, the work of Haber, Kleiber and their associates cited in the references). Now, use of operators other than the CTO can lead to significant numerical 
errors in the computed values of sensitivities. Thus, the consequences of using, for example, the continuum tangent operator, can be more serious for sensitivity analysis than for the usual analysis of the nonlinear mechanics problem.

This paper presents, for the first time, the use of the CTO for solving usual and sensitivity problems in elastoplasticity by the boundary element method. An implicit BEM scheme is proposed in which one proceeds from step $n$ to $\operatorname{step} n+1$ with the stress increment obtained from the BEM equations and the stress at step $n+1$ obtained from the radial return algorithm (RRA). As expected, the global CTO appears when the Newton method is applied to solve the usual problem, and its converged value appears in the corresponding linear sensitivity equation for that step. Numerical results are presented for pressurization of a spherical cavity in an infinite elastoplastic medium. The results for all the examples are uniformly excellent. The algorithm proposed here appears to be both robust and very powerful. Very large load steps (up to $6 p_{y}$, where $p_{y}$ is the pressure for first yielding in the body) can be: accommodated by the iterative algorithm and accurate results are obtained within a few iterations. It is also interesting to observe that, for the numerical examples presented in this paper, the final converged results are almost independent of load step size. On the other hand, as expected, for a fixed spatial grid size $\Delta r / a$, the final accuracy decreases as the final load increases. Thus, as is usual in numerical computations, the chosen mesh size must be fine enough for the problem being solved in order to assure an acceptable solution.

The approach presented in this paper appears to be extremely promising for both the usual as well as sensitivity analysis of elasto-plastic problems.

Acknowledgements-Most of the research presented was performed when S. Mukherjee was on sabbatical leave at the Laboratoire de Mécanique des Solides, Ecole Polytechnique, Palaiseau, France. S. Mukherjee also acknowledges partial financial support from a research contract from the General Motors Research Laboratories, Warren, Michigan, USA, with Cornell University.

\section{REFERENCES}

Arora, J. S. and Cardoso, J. B. (1992). Variational principle for shape sensitivity analysis. AIAA J. 30, 538-547. Badrinarayanan, S. and Zabaras, N. (1996). A sensitivity analysis for the optimal design of metal forming processes. Comp. Meth. Appl. Mech. Engng, in press.

Banerjee, P. K. (1994). The Boundary Element Method in Engineering (2nd edition), McGraw Hill, London.

Chandra, A. and Mukherjee, S. (1996). Boundary Element Methods in Manufacturing, Oxford University Press, Oxford, UK.

Choi, K. K. and Santos, J. L. T. (1987). Variational methods for design sensitivity analysis in nonlinear structural systems; part I : theory. Int. J. Num. Meth. Engng 24, 2039-2055.

Haslinger, J. and Neittaanmaki, P. (1988). Finite Element Approximation for Optimal Shape Design: Theory and Applications, John Wiley and Sons, Chichester, U.K.

Jao, S. Y. and Arora, S. J. (1992). Design sensitivity analysis of nonlinearstructures using endochronic constitutive model. Part 1: general theory. Comp. Mech. 10, 39-57.

Jao, S. Y. and Arora, S. J. (1992). Design sensitivity analysis of nonlinear structures using endochronic constitutive model. Part 2: discretization and applications. Comp. Mech. 10, 59-72.

Jin, H., Runesson, K. and Matiasson, K. (1989). Boundary element formulation in finite deformation plasticity using implicit integration. Comp. Struct. 31, 25-34.

Kleiber, M. (1991). Computational coupled non-associative thermoplasticity. Comp. Meth. Appl. Mech. Engng 90, 943-967.

Kleiber, M., Hien, T. D. and Postek, E. (1994). Incremental finite-element sensitivity analysis for non-linear mechanics applications. Int. J. Num. Meth. Engng 37, 3291-3308.

Kleiber, M., Hien, T. D., Antúnez, H. and Kowalczyk, P. (1995). Parameter sensitivity of elasto-plastic response. Engng Comput. 12, 263-280.

Lee, T. H. and Arora, J. S. (1995). A computational method for design sensitivity analysis of elastoplastic structures. Comp. Meth. Appl. Mech. Engng 122, 27-50.

Leu, L. J. and Mukherjee, S. (1993). Sensitivity analysis and shape optimization in nonlinear solid mechanics. Engng Anal. Bcund. Elem. 12, 251-260.

Leu, L. J. and Mukherjee, S. (1994). Implicit objective integration for sensitivity analysis in nonlinear solid mechanics. Int. J. Num. Meth. Engng 37, 3843-3868.

Leu, L. J. and Mukherjee, S. (1994). Sensitivity analysis of hyperelastic-viscoplastic solids undergoing large deformations. Comp. Mech. 15, 101-116.

Leu, L. J. and Mukherjee, S. (1995). Sensitivity analysis in nonlinear solid mechanics by the boundary element method with an implicit scheme. J. Engng Anal. Des. 2, 33-55.

Lubliner, J. (1990). Plasticity Theory. Macmillan, NY. 
Michaleris, P., Tortorelli, D. A. and Vidal, C. A. (1994). Tangent operators and design sensitivity formulations for transient non-linear coupled problems with applications to elastoplasticity. Int. J. Num. Meth. Engng 37, 2471-2499.

Santos, J. L. T. and Choi, K. K. (1988). Sizing design sensitivity analysis of nonlinear structural systems, part II: numerical method. Int. J. Num. Meth. Engng 26, 2097-2114.

Simo, J. C. and Taylor, R. L. (1985). Consistent tangent operators for rate-independent elastoplasticity. Comp. Meth. Appl. Mech. Engng 48, 101-118.

Telles, J. C. F. and Carrer, J. A. M. (1991). Implicit procedures for the solution of elastoplastic problems by the boundary element method. Math. Comput. Modelling 15, 303-311.

Telles, J. C. F. and Carrer, J. A. M. (1994). Static and transient dynamic nonlinear stress analysis by the boundary element method with implicit techniques. Engng Anal. Bound. Elem. 14, 65-74.

Vidal, C. A., Lee, H. S. and Haber, R. B. (1991). The consistent tangent operator for design sensitivity of historydependent response. Comp. Syst. Engng 2, 509-523.

Vidal, C. A. and Haber, R. B. (1993). Design sensitivity analysis for rate-independent elastoplasticity. Comp. Meth. Appl. Mech. Engng 107, 393-431.

Wei, X., Leu, L. J., Chandra, A. and Mukherjee, S. (1994). Shape optimization in elasticity and elastoplasticity. Int. J. Solids Structures 31, 533-550.

Zhang, Q., Mukherjee, S. and Chandra, A. (1992). Design sensitivity coefficients for elasto-viscoplastic problems by boundary element methods. Int. J. Num. Meth. Engng 34, 947-966.

Zhang, Q., Mukherjee, S. and Chandra, A. (1992). Shape design sensitivity analysis for geometrically and materially nonlinear problems by the boundary element method. Int. J. Solids Structures 29, 2503-2525.

\section{APPENDIX}

Spherical cavity in an infinite elasto-plastic medium with linear work hardening.

Exact solution

In the linear hardening case (i.e., $m=1$ ), the outer radius $b=b(\lambda)$ of the plastic region $r \in[a, b]$ for a given load $\lambda \geqslant 0$ is given by the unique solution of the transcendental equation

$$
3 \zeta \ln X^{3}+2 k_{1}\left(X^{3}-1\right)=\lambda\left(3 \zeta+2 k_{1}\right)
$$

with $X=(b / a)$ and

$$
\zeta=\frac{1+v}{3(1-v)}
$$

The analytical solution in the plastic region $a \leqslant r \leqslant a X$ is then given as follows:

$$
\begin{aligned}
u(r) & =a\left[D x+C x^{-2}+B x \ln x\right] \\
e(r) & =B-C x^{-3} \\
\bar{e}^{p}(r) & =\frac{2 k_{0}}{3 \zeta+2 k_{1}}\left[\frac{X^{3}}{x^{3}}-1\right] \quad(x=r / a)
\end{aligned}
$$

with the constants

$$
B=\frac{3(1-\zeta)}{3 \zeta+2 k_{1}} k_{0}, \quad C=\frac{3+2 k_{1}}{3 \zeta+2 k_{1}} X^{3} k_{0}, \quad D=-\frac{B}{3}\left(1+\ln X^{3}\right)
$$

In the absence of hardening, the previous solution still holds with $k_{1}=0$; moreover the plastic radius is explicitly given by

$$
X=b / a=\exp (\lambda / 3)
$$

Exact solutions for various sensitivities, e.g., with respect to the hardening parameter $k_{1}$, can finally be obtained in a straightforward way.

To the best of the authors' knowledge, no analytical solution is known for general power-law hardening, i.e., when $m \neq 0,1$ in (39).

\section{Integral representation}

The one-dimensional counterparts of eqns (19) and (20) are given below.

$$
\begin{gathered}
e(r)=3 \zeta \frac{a^{3}}{r^{3}} \int_{a}^{+\infty} e^{p}(\rho) \frac{\mathrm{d} \rho}{\rho}-k_{0}(1+\lambda) \frac{a^{3}}{r^{3}}+(1-\zeta) e^{p}(r) \\
0=3 \zeta \frac{a^{3}}{r^{3}} \int_{a}^{+\infty}\left[e-\frac{s}{2 G}\right](\rho) \frac{\mathrm{d} \rho}{\rho}-k_{0}(1+\lambda) \frac{a^{3}}{r^{3}}-\zeta e(r)-(1-\zeta) \frac{s(r)}{2 G} .
\end{gathered}
$$

